\title{
Trends in element incorporation in hyaline and porcelaneous foraminifera as a function of $p \mathrm{CO}_{2}$
}

\author{
Inge van Dijk ${ }^{1}$, Lennart J. de Nooijer ${ }^{1}$, and Gert-Jan Reichart ${ }^{1,2}$ \\ ${ }^{1}$ Department of Ocean Systems, NIOZ-Royal Netherlands Institute for Sea Research and Utrecht University, Postbus 59, \\ 1790 AB, Den Burg, the Netherlands \\ ${ }^{2}$ Faculty of Geosciences, Earth Sciences Department, Utrecht University, Budapestlaan 4, 3584 CD, Utrecht, the Netherlands \\ Correspondence to: Inge van Dijk (inge.van.dijk@nioz.nl)
}

Received: 21 September 2016 - Published in Biogeosciences Discuss.: 27 September 2016

Revised: 21 December 2016 - Accepted: 30 December 2016 - Published: 2 February 2017

\begin{abstract}
In this study we analyzed the impact of seawater carbonate chemistry on the incorporation of elements in both hyaline and porcelaneous larger benthic foraminifera. We observed a higher incorporation of $\mathrm{Zn}$ and $\mathrm{Ba}$ when $p \mathrm{CO}_{2}$ increases from 350 to $1200 \mathrm{ppm}$. Modeling the activity of free ions as a function of $p \mathrm{CO}_{2}$ shows that speciation of some elements (like $\mathrm{Zn}$ and $\mathrm{Ba}$ ) is mainly influenced by the formation of carbonate complexes in seawater. Hence, differences in foraminiferal uptake of these might be related primarily by the speciation of these elements in seawater. We investigated differences in trends in element incorporation between hyaline (perforate) and porcelaneous (imperforate) foraminifera in order to unravel processes involved in element uptake and subsequent foraminiferal calcification. In hyaline foraminifera we observed a correlation of element incorporation of different elements between species, reflected by a general higher incorporation of elements in species with higher $\mathrm{Mg}$ content. Between porcelaneous species, interelement differences are much smaller. Besides these contrasting trends in element incorporation, however, similar trends are observed in element incorporation as a function of seawater carbonate chemistry in both hyaline and porcelaneous species. This suggests similar mechanisms responsible for the transportation of ions to the site of calcification for these groups of foraminifera, although the contribution of these processes might differ across species.
\end{abstract}

\section{Introduction}

Calcareous foraminifera, cosmopolitan unicellular protists, are widely used to reconstruct past environmental conditions, since the chemical composition of the carbonate shells reflect a wide variety of environmental parameters. For instance, the $\mathrm{Mg} / \mathrm{Ca}$ of foraminiferal shells is primarily determined by seawater temperature (Nürnberg et al., 1996; Allen and Sanders, 1994) and seawater Mg / Ca (Segev and Erez, 2006; Evans et al., 2015) and has been widely applied as a paleothermometer (Elderfield and Ganssen, 2000; Lear et al., 2000). The use of foraminifera as proxies for the inorganic carbon system in the past (seawater $\mathrm{pH}$, alkalinity, saturation state, etc.) has more recently been added to the foraminiferal proxy toolbox. For example, the concentrations of trace elements in foraminiferal shells, including U (Keul et al., 2013; Russell et al., 2004), Zn (Marchitto et al., 2000; van Dijk et al., 2017) and B (Yu and Elderfield, 2007), correlate with seawater carbonate ion concentration $\left(\left[\mathrm{CO}_{3}^{2-}\right]\right)$, while the boron isotopic composition of foraminiferal calcite is used as a proxy for pH (Sanyal et al., 1996). However, insight into vital effects (Erez, 2003) and inter-specific differences in trace element incorporation (Bentov and Erez, 2006; Toyofuku et al., 2011; Wit et al., 2012) is needed to increase robustness of these proxies.

On the broadest taxonomic scale, foraminifera produce tests using either one of two fundamentally different mechanisms. These calcification strategies reflect the evolutionary separation of foraminiferal groups dating back to the Cambrian diversification, from where the imperforate porcelaneous species and perforate hyaline foraminifera developed 
independently (Pawlowski et al., 2003). Previously observed species-specific differences in partitioning and fractionation of elements most likely primarily reflect these differences in calcification strategy, since these offsets are largest between hyaline and porcelaneous species (for a summary, see Toyofuku et al., 2011). The calcification process of the latter group has been studied more extensively than that of the porcelaneous species (De Nooijer et al., 2014). Although many aspects of perforate calcification remain unsolved, there is consensus that chamber formation takes place extracellularly, but within a (semi-)enclosed space, generally termed the site of calcification (SOC). The first layers of calcite precipitate on an organic matrix (the POS or primary organic sheet) that serves as a template for the calcite layer that forms the chamber wall (Erez, 2003; Hemleben et al., 1977). To promote calcification, foraminifera furthermore need to remove $\mathrm{Mg}$ ions and/or protons (Zeebe and Sanyal, 2002) from the seawater entering the SOC. A number of larger benthic foraminifera form hyaline shells, although the amount of $\mathrm{Mg}$ in their shells is often more than 10 times higher than that of planktonic and small benthic hyaline species, hence covering a larger range in $\mathrm{Mg} / \mathrm{Ca}_{\text {CALCITE values. The calcifica- }}$ tion strategy of porcelaneous foraminifera is less well studied, which may be partly explained by their limited application in paleoceanography. Porcelaneous foraminifera use a different mode of calcification (Debenay et al., 1998; De Nooijer et al., 2009; Berthold, 1976; Hemleben et al., 1986) and produce shells without pores (hence the term imperforate) consisting of tablets or needles (Erez, 2003; Bentov and Erez, 2006; Debenay et al., 1998). These calcitic needles (2$3 \mu \mathrm{m})$ are precipitated intracellularly (Berthold, 1976), after which they are transported out of the foraminifer to form a new chamber (Angell, 1980). At the outer and inner layers of these chambers, the needles are arranged along the same orientation so that they form an optically homogenous surface, giving it a shiny (hence the term "porcelaneous") appearance. In general, the $\mathrm{Mg} / \mathrm{Ca}$ values of the shells of porcelaneous foraminifera are high.

Remarkably, despite this large biological control, incorporation of minor and trace elements still reflects environmental conditions, in both hyaline and porcelaneous foraminiferal shells. Systematic offsets between different species, interdependence of trace elements incorporated (Langer et al., 2016) and the different response of element incorporation on element speciation (Wit et al., 2013; Keul et al., 2013; van Dijk et al., 2017) potentially provide useful clues for determining which processes play an important role in the biomineralization pathways. Here we present the results from a controlled growth experiment for which we used several (intermediateand high-Mg) hyaline and porcelaneous species combined with an inter-species comparison of trace element incorporation. We assessed the impact of bio-calcification on element incorporation as a function of $p \mathrm{CO}_{2}$ in order to explore the proposed inorganic carbonate proxies (e.g., $\mathrm{Zn} / \mathrm{Ca}$; van Dijk et al., 2017) and the impact of different calcification strate- gies (hyaline versus porcelaneous) on element partitioning. We cultured eight benthic foraminiferal species (four hyaline and four porcelaneous) under four different $p \mathrm{CO}_{2}$ conditions, analyzing incorporation of $\mathrm{Mg}, \mathrm{Sr}, \mathrm{Na}, \mathrm{Zn}$ and $\mathrm{Ba}$.

\section{Methods}

\subsection{Foraminiferal collection}

Large samples of macroalgae (Dictyota sp.) were collected in November 2015 at a depth of $2-3 \mathrm{~m}$ in Gallows Bay, St. Eustatius $\left(17^{\circ} 28^{\prime} 31.6^{\prime \prime} \mathrm{N}, 62^{\circ} 59^{\prime} 9.4^{\prime \prime} \mathrm{W}\right)$. Salinity was $\sim 34$ and temperature was $\sim 29^{\circ} \mathrm{C}$ at the site of collection. The collected macroalgae were transported to the laboratory at the Caribbean Netherlands Science Institute (CNSI), where they were placed in a $5 \mathrm{~L}$ aquarium with aerated and unfiltered seawater. From this stock, small amounts of algae and debris were gently sieved over a 90 and $600 \mu \mathrm{m}$ mesh to carefully dislodge foraminifera. Several species of foraminifera were picked from the resulting $90-600 \mu \mathrm{m}$ fraction and directly from the macroalgae. Living specimens of Sorites marginalis Lamarck, 1816, Amphistegina gibbosa d'Orbigny, 1839, Laevipeneroplis bradyi Cushman, 1930 and Archaias angulatus Fichtel and Moll, 1798 and limited amounts $(<20)$ of Peneroplis pertusus Forskål, 1775, Asterigerina carinata d'Orbigny, 1839, Heterostegina antillarum d'Orbigny, 1839, and Planorbulina acervalis Brady, 1884, characterized by yellow cytoplasm and pseudopodial activity, were selected for the culturing experiments.

\subsection{Culture setup}

Four barrels, each filled with $100 \mathrm{~L}$ of seawater $(5 \mu \mathrm{m}$ filtered), were connected to a $\mathrm{Li}-\mathrm{Cor} \mathrm{CO}_{2} / \mathrm{H}_{2} \mathrm{O}$ analyzer (LI7000) to regulate the $\mathrm{CO}_{2}$ level in the barrels' headspace. The set points were maintained by addition of $\mathrm{CO}_{2}$ and/or $\mathrm{CO}_{2}$-scrubbed air according to the monitored $p \mathrm{CO}_{2}$. The set points for $p \mathrm{CO}_{2}$ were $350,450,760$ and $1400 \mathrm{ppm}$, resulting in four batches of seawater (treatments A-D) differing only in their inorganic carbon chemistry. Salinity $(34.0 \pm 0.2)$ was monitored with a salinometer (VWR CO310). The fluorescent compound calcein (bis $[N, N$ bis(carboxymethyl)aminomethyl]-fluorescein) was added to the culture media ( $5 \mathrm{mg} \mathrm{L}^{-1}$ seawater) to enable determination of newly formed chambers during the culture experiment (Bernhard et al., 2004). Short-term exposure ( $<3$ weeks) to calcein has no detectable impact on the physiology of benthic foraminifera (Kurtarkar et al., 2015), and the presence of calcein has no effect on the incorporation of $\mathrm{Mg}$ and $\mathrm{Sr}$ in foraminiferal calcite (Dissard et al., 2009). Culture media were stored air-free in portions of $250 \mathrm{~mL}$ in Nalgene bottles with Teflon-lined caps at $4{ }^{\circ} \mathrm{C}$ until further use. 


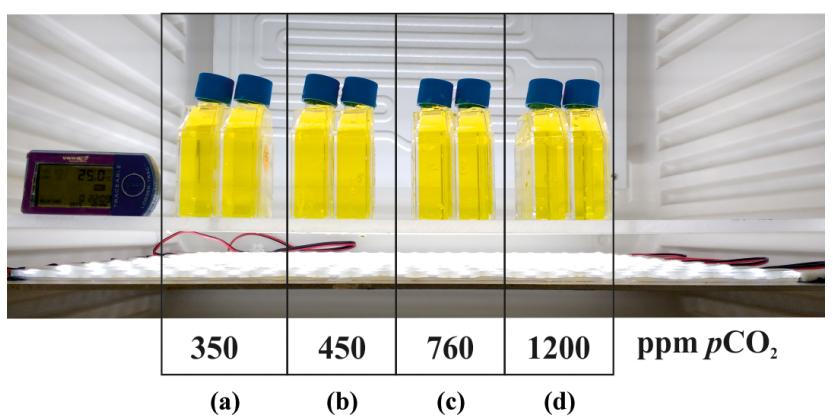

Figure 1. Photograph of the culture setup. Four duplicate bottles with culture media (with calcein added) and adult specimens of foraminifera. Treatments with corresponding set points are A, 350 ppm; B, 450 ppm; C, 760 ppm; D, 1200 ppm $\mathrm{CO}_{2}$.

Foraminifera were divided over the different treatments in duplicate and placed in $70 \mathrm{~mL}$ Falcon ${ }^{\circledR}$ tissue bottles with gas-tight caps in a thermostat set at $25^{\circ} \mathrm{C}$ (Fig. 1). The thermostat was monitored by a temperature logger (Traceable Logger Trac, Maxi Thermal), recording the temperature every minute. The average temperature over the whole experiment was $25 \pm 0.2^{\circ} \mathrm{C}$. To create uniform light conditions, the thermostat was equipped with two LED shelves, which resulted in high light conditions $12 \mathrm{~h} / 12 \mathrm{~h}$. Culture media were replaced every four days to avoid build-up of organic waste and to obtain stable seawater element concentrations and carbon chemistry. Foraminifera were fed after every water change with $0.5 \mathrm{~mL}$ of concentrated freeze-dried Dunaliella salina cells, pre-diluted with the corresponding treatment seawater. After 21 days, the experiment was terminated. Foraminifera were rinsed three times with deionized water, dried at $40{ }^{\circ} \mathrm{C}$ and stored in micropaleontology slides until further analysis at the Royal Netherlands Institute for Sea Research (NIOZ).

\subsection{Analytical methods}

\subsubsection{Seawater carbon parameters}

At the start and termination of the experiment, $125 \mathrm{~mL}$ samples of the seawater at each of the different experimental conditions were collected to analyze dissolved inorganic carbon (DIC) and total alkalinity (TA) on a Versatile INstrument for the Determination of Titration Alkalinity (VINDTA) at the CNSI. Using the measured DIC and TA values and the software CO2SYS v2.1, adapted to Excel by Pierrot et al. (2006), the other carbon parameters (including $\left[\mathrm{CO}_{3}^{2-}\right]$ and $\Omega_{\text {CALCITE }}$ ) were calculated. For this we used the equilibrium constants for $\mathrm{K} 1$ and $\mathrm{K} 2$ from Lueker et al. (2000) and $\mathrm{KHSO}_{4}$ from (Dickson, 1990) (Table 1).

\subsubsection{Seawater element concentrations}

At the start and end of the experiment and during replacement of the culture media, subsamples were collected in duplicate using $50 \mathrm{~mL}$ LDPE Nalgene bottles and immediately frozen at $-80^{\circ} \mathrm{C}$. After transportation to the NIOZ, defrosted samples were acidified with triple quartz-distilled $\mathrm{HCl}$ to $\mathrm{pH} \sim 1.8$, and the seawater composition of the samples was analyzed on an Element 2 sector field double-focusing mass spectrometer (SF-ICP-MS) run in medium-resolution mode. IAPSO (International Association for the Physical Sciences of the Ocean) standard seawater was used as a drift monitor. Analytical precision (relative standard deviation) was $3 \%$ for $\mathrm{Ca}, 4 \%$ for $\mathrm{Mg}, 1 \% \mathrm{Na}, 1 \%$ for $\mathrm{Sr}$ and $5 \% \mathrm{Ba}$. We obtained average values of $5.25 \pm 0.06 \mathrm{~mol} \mathrm{~mol}^{-1}$ for $\mathrm{Mg} / \mathrm{Ca}$, $44.6 \pm 0.6 \mathrm{~mol} \mathrm{~mol}^{-1}$ for $\mathrm{Na} / \mathrm{Ca}, 8.63 \pm 0.05 \mathrm{~mol} \mathrm{~mol}^{-1}$ for $\mathrm{Sr} / \mathrm{Ca}$, and $9.04 \pm 0.47 \mu \mathrm{mol} \mathrm{mol}^{-1}$ for $\mathrm{Ba} / \mathrm{Ca}$.

A subsample was analyzed using a commercially available pre-concentration system, SeaFAST S2. With the SeaFAST system, elements with low concentrations are preconcentrated to values above detection limit of the SF-ICPMS. Accordingly, we measured Cd, Pb, U, B, Ti, Mn, Fe, Co, $\mathrm{Ni}, \mathrm{Cu}$, and $\mathrm{Zn}$. In short, $10 \mathrm{~mL}$ of sample was mixed with an ammonium acetate buffer to pH 6.2 and loaded on a column containing NOBIAS chelating agent. After rinsing the column with a diluted ammonium acetate buffer the metals were eluted in $750 \mu \mathrm{L}$ of quartz-distilled $1.5 \mathrm{M} \mathrm{HNO}_{3}$ before being quantified on the SF-ICP-MS. Here we use the Zn data only, as this was analyzed in the foraminifera as well. Analytical precision (relative standard deviation) was $5 \%$ for $\mathrm{Zn}$. We obtained an average value of $15.3 \pm 0.5 \mu \mathrm{mol} \mathrm{mol}^{-1}$ for seawater $\mathrm{Zn} / \mathrm{Ca}$ for all treatments. Although these values are clearly above natural open ocean values, the concentrations are very uniform between treatments and when comparing start and end of the experiments. The contamination with $\mathrm{Zn}$ hence might have already occurred when filling the culture setup with the waters from the bay adjacent to the culture facility. Concentrations are well below values considered harmful for foraminifera (Nardelli et al., 2016).

\subsubsection{Cleaning methods}

After termination of the experiment, foraminiferal shells were cleaned following an adapted version of Barker et al. (2003). Per treatment duplicate, all foraminifera were transferred to $10 \mathrm{~mL}$ polyethylene vials. To each vial, $10 \mathrm{~mL}$ of $1 \% \mathrm{H}_{2} \mathrm{O}_{2}$ solution (buffered with $0.5 \mathrm{M} \mathrm{NH} \mathrm{NH}_{4} \mathrm{OH}$ ) was added to remove organic matter. The vials were heated for $10 \mathrm{~min}$ in a water bath at $95^{\circ} \mathrm{C}$, and placed in an ultrasonic bath for $30 \mathrm{~s}$ (degas mode, $80 \mathrm{kHz}, 50 \%$ power), after which the oxidizing reagent was removed. These steps (organic removal procedure) were repeated five times. Foraminiferal samples were rinsed five times with ultrapure water, after which the vials were stored overnight in a laminar flow cabinet at room temperature to dry. Dried foraminifera were 
Table 1. Carbon parameters (TA ,total alkalinity, $n=2$; DIC, dissolved inorganic carbon, $n=2$ ) with (relative) standard deviation of the culture water per treatment of the $p \mathrm{CO}_{2}$ experiment. CO2SYS was used to calculate seawater carbonate ion concentration, calcite saturation state and $\mathrm{pH}$ from measured TA and DIC.

\begin{tabular}{|c|c|c|c|c|c|c|}
\hline \multirow{2}{*}{ 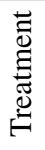 } & \multirow{2}{*}{$\begin{array}{l}\text { Set point } \\
\qquad \mathrm{CO}_{2} \\
\text { ppm }\end{array}$} & \multicolumn{2}{|c|}{ Measured } & \multicolumn{3}{|c|}{ Calculated CO2SYS } \\
\hline & & $\begin{array}{r}\text { TA } \\
\mu \mathrm{mol} \mathrm{kg}\end{array}$ & $\begin{array}{r}\text { DIC } \\
\mu \mathrm{mol} \mathrm{kg}\end{array}$ & $\begin{array}{r}{\left[\mathrm{CO}_{3}^{2-}\right]} \\
\mu \mathrm{mol} \mathrm{kg}-1\end{array}$ & $\begin{array}{r}\mathrm{pH} \\
(\text { total scale) }\end{array}$ & $\Omega_{\text {CALCITE }}$ \\
\hline A & 350 & $2302.8 \pm 8.2$ & $2007.5 \pm 10.7$ & 220.7 & 8.06 & 5.4 \\
\hline B & 450 & $2305.2 \pm 5.8$ & $2021.3 \pm 12.5$ & 200.0 & 8.01 & 4.9 \\
\hline $\mathrm{C}$ & 760 & $2304.4 \pm 0.9$ & $2100.8 \pm 13.4$ & 153.7 & 7.87 & 3.7 \\
\hline $\mathrm{D}$ & 1200 & $2300.3 \pm 0.7$ & $2201.4 \pm 4.1$ & 92.2 & 7.61 & 2.2 \\
\hline
\end{tabular}
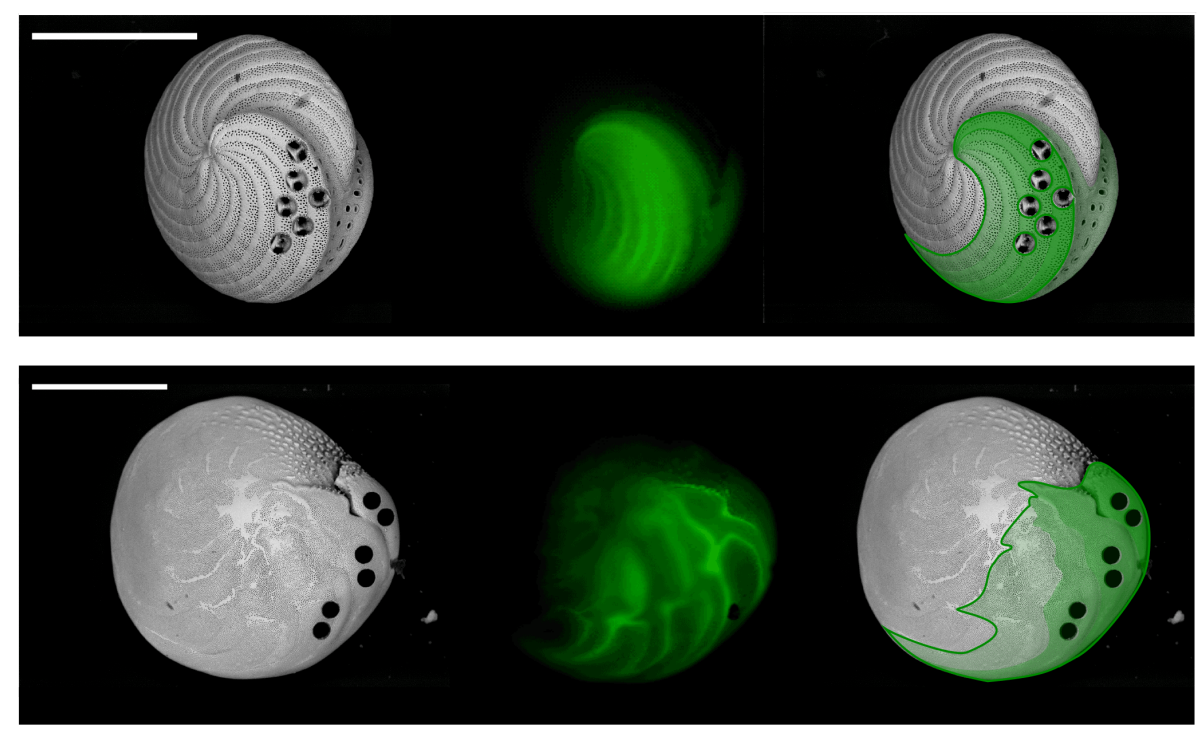

Figure 2. SEM (left) and fluorescence microscope (middle) photographs of A. angulatus (top series) and A. gibbosa (bottom series) to assess newly formed chambers for laser ablation (right). Scale bar $=500 \mu \mathrm{m}$.

placed on double-sided tape on LA-ICP-MS stubs. Pictures were taken of individual foraminifera with a ZEISS Axioplan 2 fluorescence microscope equipped with appropriate excitation and emission optics and a ZEISS Axiocam MRc 5 camera to assess the number of chambers added during the experiment based on the incorporation of calcein.

\subsubsection{LA-ICP-MS}

Element concentrations of individual fluorescent chambers were analyzed by laser ablation ICP-MS (Reichart et al., 2003; van Dijk et al., 2017). To determine foraminiferal element concentrations, the laser system (NWR193UC, New Wave Research) at the Royal NIOZ was equipped with a twovolume sample cell (New Wave Research), characterized by a wash-out time of $1.8 \mathrm{~s}$ ( $1 \%$ level) and hence allowing detection of variability in obtained element to $\mathrm{Ca}$ ratios within chamber walls. Single chambers were ablated in a helium environment using a circular laser spot with a diameter of $80 \mu \mathrm{m}$ (S. marginalis) or $60 \mu \mathrm{m}$ (other species). We ablated all calcein-stained chambers twice, except for the first 1-2 chambers that formed during the experiment to avoid contamination of calcite of chambers formed prior to the experiments and overlapped by the labeled chambers (Fig. 2).

All foraminiferal samples were ablated with an energy density of $1 \pm 0.1 \mathrm{~J} \mathrm{~cm}^{-2}$ and a repetition rate of $6 \mathrm{~Hz}$. The resulting aerosol was transported on a helium flow through an in-house-built smoothing device, being mixed with a nitrogen flow $\left(5 \mathrm{~mL} \mathrm{~min}^{-1}\right)$ before entering the quadrupole ICP-MS (iCAP-Q, Thermo Scientific). Monitored masses included ${ }^{7} \mathrm{Li},{ }^{11} \mathrm{~B},{ }^{23} \mathrm{Na},{ }^{24} \mathrm{Mg},{ }^{25} \mathrm{Mg},{ }^{27} \mathrm{Al},{ }^{43} \mathrm{Ca},{ }^{44} \mathrm{Ca},{ }^{66} \mathrm{Zn}$, ${ }^{88} \mathrm{Sr}$ and ${ }^{137} \mathrm{Ba}$. Contrary to ${ }^{67} \mathrm{Zn}$ and ${ }^{68} \mathrm{Zn},{ }^{66} \mathrm{Zn}$ is free of interferences when measuring calcium carbonate and SRM NIST (National Institute of Standard and Technology, USA, standard reference material) glass standards (Jochum et al., 2012). Potential contamination or diagenesis of the outer or inner layer of calcite was excluded by monitoring the Al signal. At the start of each series, we analyzed SRM NIST612 and NIST610 glass standard in triplicate (using an energy 
Table 2. Total number of LA-ICP-MS measurements per species, per treatment (A-D).

\begin{tabular}{lrrrr}
\hline & \multicolumn{4}{c}{$n$ measurements (n specimens) } \\
\cline { 2 - 5 } Species & A: $350 \mathrm{ppm}$ & $\mathrm{B}: 450 \mathrm{ppm}$ & C: $760 \mathrm{ppm}$ & D: $1200 \mathrm{ppm}$ \\
\hline A. angulatus & $62(19)$ & $72(21)$ & $76(21)$ & $51(14)$ \\
S. marginalis & $48(14)$ & $49(15)$ & $57(18)$ & $33(11)$ \\
A. gibbosa & $106(28)$ & $126(32)$ & $75(18)$ & $59(15)$ \\
L. bradyi & $21(5)$ & $38(13)$ & $27(5)$ & $16(4)$ \\
A. carinata & $12(2)$ & $14(1)$ & $19(4)$ & $5(1)$ \\
P. pertusus & $12(2)$ & $11(2)$ \\
H. antillarum & \multicolumn{5}{c}{$14(2)$} \\
P. acervalis & $187(49)$ & $331(87)$ & $254(66)$ \\
\hline Total & \multicolumn{5}{c}{$189(49)$} \\
\hline
\end{tabular}

density of $5 \pm 0.1 \mathrm{~J} \mathrm{~cm}^{-2}$ ), JCt-1 (coral carbonate) and two in-house standards, namely NFHS (NIOZ foraminifera house standard; Mezger et al., 2016) and the Iceland spar NCHS (NIOZ calcite house standard). We further analyzed JCp-1 (coral, Porites sp.; Okai et al., 2002) and MACS-3 (synthetic calcium carbonate) at the start of each series and monitored drift after every 10 samples. All element to calcium ratios were calculated with an adapted version of the MATLABbased program SILLS (Signal Integration for Laboratory Laser Systems; Guillong et al., 2008). SILLS was modified by NIOZ to evaluate LA-ICP-MS measurements on foraminifera, allowing import of Thermo Qtegra software sample list, laser data reduction and laser LOG files. Major adaptations include improved automated integration and evaluation of (calibration and monitor) standards, quality control report of the monitor standards and export in element to calcium ratios $\left(\mathrm{mmol} \mathrm{mol}^{-1}\right)$. Calibration was performed against the MACS- 3 carbonate standard, with ${ }^{43} \mathrm{Ca}$ as an internal standard, and we used the multiple measurements of MACS-3 for a linear drift correction. Relative analytical precision (relative standard deviation (RSD) of all MACS-3 analyses) is $3 \%$ for ${ }^{23} \mathrm{Na}, 3 \%$ for ${ }^{24} \mathrm{Mg}, 3 \%$ for ${ }^{25} \mathrm{Mg}, 4 \%$ for ${ }^{66} \mathrm{Zn}, 3 \%$ for ${ }^{88} \mathrm{Sr}$ and $3 \%$ for ${ }^{137} \mathrm{Ba}$. In total, 961 analyses were performed on 251 specimens covering eight species cultured in four experimental conditions (see Table 2 for details).

We calculated the standard deviation (SD), RSD and standard error $(\mathrm{SD} / \sqrt{ } \mathrm{n} ; \mathrm{SE})$ per treatment. The partitioning coefficient $(D)$ of an element $(E)$ between seawater and foraminiferal calcite is expressed as $D_{\mathrm{E}}=(E / \mathrm{Ca}$ CALCiTe $) /(E / \mathrm{Casw})$. Partition coefficients and element versus calcium ratio parameters were statistically compared with different experimental parameters (such as $p \mathrm{CO}_{2}$ or $\left.\left[\mathrm{CO}_{3}^{2-}\right]\right)$ using a two-sided $t$ test with $95 \%$ confidence levels. This also allows for the calculation of $95 \%$ confidence intervals over the average per treatment. Pairwise comparisons were made for per $E$ / Ca per species and culture conditions using ANOVA. Groups that showed significant difference were assigned different letters. When com- paring partition coefficients to other studies, $E$ / Casw data were, in some studies, not measured. For these studies we used average seawater $E / C$ asw to calculate $D_{\mathrm{E}}$ (see also Table S1 in the Supplement), allowing comparison of partitioning coefficients.

\section{Results}

\subsection{Element / Ca as a function of ocean acidification}

In hyaline species $\mathrm{Mg} / \mathrm{Ca}$ CALCITE varies between 25.9 and $141.3 \mathrm{mmol} \mathrm{mol}^{-1} \mathrm{Mg} / \mathrm{Ca}$. In contrast, $\mathrm{Mg} / \mathrm{Ca}$ CALCITE of porcelaneous species ranges from 121.3 to $149.3 \mathrm{mmol} \mathrm{mol}^{-1}$ (Table 3). This large spread in foraminifera $E / \mathrm{Ca}$ of hyaline species is also observed for $\mathrm{Sr}\left(1.7-3.1 \mathrm{mmol} \mathrm{mol}^{-1}\right)$, Na $\left(3.4-19.5 \mathrm{mmol} \mathrm{mol}^{-1}\right)$, Zn $\left(9.0-97.0 \mu \mathrm{mol} \mathrm{mol}^{-1}\right)$ and Ba $\left(2.7-20.1 \mu \mathrm{mol} \mathrm{mol}^{-1}\right)$, while porcelaneous species only vary over a narrow range $\left(\mathrm{Sr}=2.0-2.2 \mathrm{mmol} \mathrm{mol}^{-1} ; \quad \mathrm{Na}=3.8-5.8 \mathrm{mmol} \mathrm{mol}^{-1}\right.$; $\mathrm{Zn}=53.0-140.8 \mu \mathrm{mol} \mathrm{mol}^{-1} ; \mathrm{Ba}=18.0-29.0 \mu \mathrm{mol} \mathrm{mol}^{-1}$ ).

In both porcelaneous and hyaline species we find an increase in $\mathrm{Zn} / \mathrm{Ca}$ CALCite and $\mathrm{Ba} / \mathrm{Ca}$ CALCITE with $p \mathrm{CO}_{2}$, while foraminiferal $\mathrm{Sr} / \mathrm{Ca}, \mathrm{Mg} / \mathrm{Ca}$ and $\mathrm{Na} / \mathrm{Ca}$ remain similar across the experimental conditions (Fig. 3 and Table 4). Sensitivity of both foraminiferal $\mathrm{Zn} / \mathrm{Ca}$ and $\mathrm{Ba} / \mathrm{Ca}$ to changes in seawater $p \mathrm{CO}_{2}$ differs between the studied porcelaneous and hyaline species. When $p \mathrm{CO}_{2}$ changes from 350 to $1200 \mathrm{ppm}, \mathrm{Zn} / \mathrm{Ca}$ of hyaline foraminifera increases by a factor of 3.7 (A. carinata) or 4.5 (A. gibbosa), while porcelaneous foraminiferal $\mathrm{Zn} / \mathrm{Ca}$ increases only by $1.3(S$. marginalis), 1.8 (A. angulatus) and 2.1 (L. bradyi). Sensitivity of foraminiferal $\mathrm{Ba} / \mathrm{Ca}$ to the same change in $p \mathrm{CO}_{2}$ also shows a similar pattern, with $\mathrm{Ba} / \mathrm{Ca}$ of hyaline species increasing by a factor of 3.6 (A. carinata) or 3.7 (A. gibbosa), while porcelaneous species increase $\mathrm{Ba} / \mathrm{Ca}$ only by a factor of 1.8 (S. marginalis), 1.6 (A. angulatus) or 2.1 (L. bradyi). 
Table 3. Overview of element to Ca ratios in foraminiferal calcite (Avg, average; SE, standard error) and partition coefficients $D_{\mathrm{E}}$, with $D_{\mathrm{E}}$ of ambient conditions (treatment B) in bold.

\begin{tabular}{|c|c|c|c|c|c|c|c|c|c|c|c|}
\hline \multirow{2}{*}{$\begin{array}{l}\frac{\mathscr{O}}{\mathscr{\delta}} \\
\text { है } \\
\text { की }\end{array}$} & \multirow{2}{*}{ 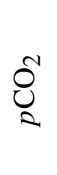 } & \multicolumn{2}{|c|}{$\begin{array}{c}\mathrm{Mg} / \mathrm{Ca} \\
\mathrm{mmol} \mathrm{mol}^{-1}\end{array}$} & \multicolumn{2}{|c|}{$\begin{array}{c}\mathrm{Na} / \mathrm{Ca} \\
\mathrm{mmol} \mathrm{mol}^{-1}\end{array}$} & \multicolumn{2}{|c|}{$\begin{array}{c}\mathrm{Sr} / \mathrm{Ca} \\
\mathrm{mmol} \mathrm{mol}^{-1}\end{array}$} & \multicolumn{2}{|c|}{ 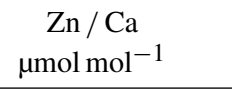 } & \multicolumn{2}{|c|}{ 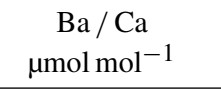 } \\
\hline & & $\operatorname{Avg} \pm \mathrm{SE}$ & $\begin{array}{r}D_{\mathrm{Mg}} \\
\times 10^{-3}\end{array}$ & $\operatorname{Avg} \pm \mathrm{SE}$ & $\begin{array}{r}D_{\mathrm{Na}} \\
\times 10^{-3}\end{array}$ & $\operatorname{Avg} \pm \mathrm{SE}$ & $D_{\mathrm{Sr}}$ & $\operatorname{Avg} \pm \mathrm{SE}$ & $D_{\mathrm{Zn}}$ & $\mathrm{Avg} \pm \mathrm{SE}$ & $D_{\mathrm{Ba}}$ \\
\hline
\end{tabular}

\begin{tabular}{|c|c|c|c|c|c|c|c|c|c|c|c|}
\hline \multicolumn{12}{|c|}{ Porcelaneous species } \\
\hline \multirow{4}{*}{ 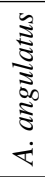 } & 350 & $139.4 \pm 0.6^{\mathrm{a}}$ & 26.6 & $5.2 \pm 0.1^{\mathrm{a}}$ & 0.12 & $2.2 \pm 0.02^{\mathrm{a}}$ & 0.25 & $80.0 \pm 5.1^{\mathrm{a}}$ & 5.3 & $13.2 \pm 0.5^{\mathrm{a}}$ & 1.5 \\
\hline & 450 & $137.7 \pm 0.5^{\mathrm{b}}$ & 26.3 & $4.3 \pm 0.1^{b}$ & 0.10 & $2.2 \pm 0.01^{\mathrm{a}}$ & 0.26 & $88.1 \pm 5.2^{b}$ & 5.8 & $14.6 \pm 0.5^{b}$ & 1.6 \\
\hline & 760 & $137.4 \pm 0.7^{b}$ & 26.2 & $4.9 \pm 0.1^{\mathrm{c}}$ & 0.11 & $2.2 \pm 0.01^{\mathrm{a}}$ & 0.26 & $122.6 \pm 7.0^{\mathrm{c}}$ & 8.1 & $17.0 \pm 0.6^{\mathrm{b}}$ & 1.9 \\
\hline & 1200 & $138.6 \pm 1.1^{\mathrm{a}}$ & 26.4 & $5.4 \pm 0.2^{\mathrm{a}}$ & 0.12 & $2.2 \pm 0.02^{\mathrm{a}}$ & 0.26 & $140.8 \pm 9.9^{\mathrm{d}}$ & 9.3 & $20.9 \pm 0.2^{\mathrm{c}}$ & 2.3 \\
\hline \multirow{4}{*}{ 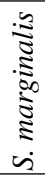 } & 350 & $147.7 \pm 0.6^{\mathrm{a}}$ & 28.2 & $4.8 \pm 0.1^{\mathrm{a}}$ & 0.11 & $2.0 \pm 0.01^{\mathrm{a}}$ & 0.24 & $70.0 \pm 10.1^{\mathrm{a}}$ & 4.6 & $17.0 \pm 0.5^{\mathrm{a}}$ & 1.9 \\
\hline & 450 & $144.2 \pm 0.8^{b}$ & 27.5 & $4.1 \pm 0.1^{b}$ & 0.09 & $2.0 \pm 0.01^{\mathrm{a}}$ & 0.23 & $74.0 \pm 10.6^{b}$ & 4.9 & $23.1 \pm 0.5^{b}$ & 2.6 \\
\hline & 760 & $143.0 \pm 0.6^{\mathrm{a}}$ & 27.3 & $3.8 \pm 0.1^{\mathrm{a}}$ & 0.09 & $2.0 \pm 0.01^{\mathrm{a}}$ & 0.23 & $87.7 \pm 15.5^{\mathrm{c}}$ & 5.8 & $27.9 \pm 0.6^{\mathrm{c}}$ & 3.1 \\
\hline & 1200 & $148.3 \pm 0.5^{\mathrm{b}}$ & 28.3 & $4.5 \pm 0.2^{\mathrm{c}}$ & 0.10 & $2.0 \pm 0.01^{\mathrm{a}}$ & 0.23 & $115.6 \pm 15.3^{\mathrm{d}}$ & 7.6 & $30.1 \pm 0.2^{\mathrm{d}}$ & 3.3 \\
\hline \multirow{4}{*}{ 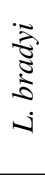 } & 350 & $137.8 \pm 1.3^{\mathrm{a}}$ & 26.3 & $5.2 \pm 0.2^{\mathrm{c}}$ & 0.12 & $2.1 \pm 0.03^{\mathrm{a}}$ & 0.24 & $60.0 \pm 6.5^{\mathrm{a}}$ & 4.0 & $14.0 \pm 0.5^{\mathrm{a}}$ & 1.5 \\
\hline & 450 & $136.2 \pm 0.7^{\mathrm{a}}$ & 26.0 & $4.3 \pm 0.1^{b}$ & 0.10 & $2.2 \pm 0.01^{b}$ & 0.25 & $73.8 \pm 6.0^{b}$ & 4.9 & $14.2 \pm 0.5^{\mathrm{a}}$ & 1.6 \\
\hline & 760 & $134.4 \pm 1.2^{\mathrm{b}}$ & 25.6 & $3.4 \pm 0.1^{\mathrm{a}}$ & 0.08 & $2.0 \pm 0.02^{\mathrm{c}}$ & 0.24 & $97.5 \pm 9.4^{\mathrm{c}}$ & 6.4 & $18.5 \pm 0.6^{\mathrm{b}}$ & 2.1 \\
\hline & 1200 & $136.9 \pm 1.1^{\mathrm{a}}$ & 26.1 & $6.2 \pm 0.2^{\mathrm{d}}$ & 0.14 & $2.1 \pm 0.02^{\mathrm{a}}$ & 0.24 & $124.2 \pm 7.8^{\mathrm{d}}$ & 8.2 & $28.8 \pm 0.2^{c}$ & 3.2 \\
\hline \multirow{3}{*}{ 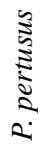 } & 350 & & & & & & & & & & \\
\hline & $\begin{array}{l}\mathbf{4 5 0} \\
760\end{array}$ & $126.1 \pm 1.8^{a}$ & 24.0 & $5.2 \pm 0.3^{\mathrm{a}}$ & 0.12 & $2.1 \pm 0.07^{a}$ & 0.25 & $53.0 \pm 10.8^{a}$ & 3.5 & $18.0 \pm 0.5^{\mathrm{a}}$ & 2.0 \\
\hline & 1200 & $121.3 \pm 1.0^{\mathrm{a}}$ & 23.1 & $5.8 \pm 0.2^{\mathrm{a}}$ & 0.13 & $2.2 \pm 0.02^{\mathrm{a}}$ & 0.26 & $75.5 \pm 11.9^{\mathrm{b}}$ & 5.0 & $29.8 \pm 0.2^{b}$ & 3.3 \\
\hline
\end{tabular}

Hyaline species

\begin{tabular}{|c|c|c|c|c|c|c|c|c|c|c|c|}
\hline 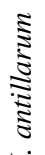 & $\begin{array}{l}350 \\
\mathbf{4 5 0} \\
760\end{array}$ & $141.3 \pm 0.3^{\mathrm{a}}$ & 26.9 & $19.4 \pm 0.5^{\mathrm{a}}$ & 0.44 & $2.7 \pm 0.02^{\mathrm{a}}$ & 0.31 & $36.0 \pm 14.7^{a}$ & 2.4 & $10.7 \pm 0.5^{\mathrm{a}}$ & 1.2 \\
\hline$\Sigma$ & 1200 & $136.9 \pm 1.6^{\mathrm{a}}$ & 26.1 & $19.5 \pm 0.4^{\mathrm{a}}$ & 0.44 & $2.7 \pm 0.02^{\mathrm{a}}$ & 0.31 & $97.0 \pm 18.3^{b}$ & 6.4 & $20.1 \pm 0.2^{\mathrm{b}}$ & 2.2 \\
\hline 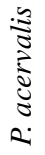 & $\begin{array}{r}350 \\
450 \\
760 \\
1200\end{array}$ & $139.1 \pm 1.2$ & 26.5 & $19.5 \pm 0.7$ & 0.46 & $3.1 \pm 0.02$ & 0.36 & $31.6 \pm 6.6$ & 2.1 & $11.3 \pm 0.5$ & 1.3 \\
\hline 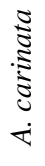 & $\begin{array}{r}350 \\
450 \\
760 \\
1200\end{array}$ & $\begin{array}{r}23.6 \pm 1.5^{\mathrm{a}} \\
\mathbf{2 8 . 5} \pm 2.4^{\mathrm{b}} \\
33.1 \pm 1.2^{\mathrm{b}} \\
33.5 \pm 3.1^{\mathrm{b}}\end{array}$ & $\begin{array}{l}4.5 \\
\mathbf{5 . 4} \\
6.3 \\
6.4\end{array}$ & $\begin{array}{r}9.9 \pm 0.4^{\mathrm{a}} \\
\mathbf{1 0 . 8} \pm \mathbf{0 . 1} \mathbf{a}^{\mathrm{a}} \\
10.9 \pm 0.2^{\mathrm{a}} \\
10.6 \pm 0.5^{\mathrm{a}}\end{array}$ & $\begin{array}{l}0.22 \\
\mathbf{0 . 2 4} \\
0.24 \\
0.24\end{array}$ & $\begin{array}{r}1.8 \pm 0.02^{\mathrm{a}} \\
\mathbf{1 . 9} \pm \mathbf{0 . 0 1} 1^{\mathrm{a}} \\
1.8 \pm 0.01^{\mathrm{a}} \\
1.8 \pm 0.03^{\mathrm{a}}\end{array}$ & $\begin{array}{l}0.21 \\
\mathbf{0 . 2 2} \\
0.21 \\
0.21\end{array}$ & $\begin{array}{r}9.0 \pm 2.6^{\mathrm{a}} \\
\mathbf{1 0 . 9} \pm \mathbf{5 . 5}^{\mathrm{a}} \\
30.7 \pm 7.0^{\mathrm{b}} \\
46.4 \pm 2.1^{\mathrm{b}}\end{array}$ & $\begin{array}{l}0.6 \\
\mathbf{0 . 7} \\
2.0 \\
3.1\end{array}$ & $\begin{array}{r}3.2 \pm 0.5^{\mathrm{a}} \\
\mathbf{6 . 0} \pm 0 . \mathbf{0 . 5}^{\mathrm{b}} \\
8.5 \pm 0.6^{\mathrm{c}} \\
11.4 \pm 0.2^{\mathrm{d}}\end{array}$ & $\begin{array}{l}0.4 \\
\mathbf{0 . 7} \\
0.9 \\
1.3\end{array}$ \\
\hline $\begin{array}{l}0 \\
\vdots \\
\vdots \\
\vdots \\
\dot{0} \\
\dot{\pi}\end{array}$ & $\begin{array}{r}350 \\
450 \\
760 \\
1200\end{array}$ & $\begin{array}{r}27.8 \pm 0.5^{\mathrm{a}} \\
\mathbf{2 5 . 9} \pm \mathbf{0 . 6}^{\mathrm{b}} \\
28.2 \pm 0.7^{\mathrm{a}} \\
28.7 \pm 0.6^{\mathrm{a}}\end{array}$ & $\begin{array}{l}5.3 \\
4.9 \\
5.4 \\
5.5\end{array}$ & $\begin{array}{r}9.0 \pm 0.1^{\mathrm{a}} \\
\mathbf{9 . 2} \pm \mathbf{0 . 1}^{\mathrm{a}} \\
9.7 \pm 0.1^{\mathrm{b}} \\
9.6 \pm 0.1^{\mathrm{b}}\end{array}$ & $\begin{array}{l}0.20 \\
\mathbf{0 . 2 1} \\
0.22 \\
0.21\end{array}$ & $\begin{array}{r}1.7 \pm 0.01^{\mathrm{a}} \\
\mathbf{1 . 7} \pm \mathbf{0 . 0 2}^{\mathrm{a}} \\
1.7 \pm 0.02^{\mathrm{a}} \\
1.7 \pm 0.02^{\mathrm{a}}\end{array}$ & $\begin{array}{l}0.20 \\
\mathbf{0 . 2 0} \\
0.20 \\
0.20\end{array}$ & $\begin{array}{r}19.0 \pm 1.8^{\mathrm{a}} \\
\mathbf{2 1 . 5} \pm \mathbf{2 . 5 ^ { \mathrm { b } }} \\
52.8 \pm 6.1^{\mathrm{c}} \\
85.8 \pm 11.3^{\mathrm{d}}\end{array}$ & $\begin{array}{l}1.3 \\
1.4 \\
3.5 \\
5.7\end{array}$ & $\begin{array}{r}2.7 \pm 0.5^{\mathrm{a}} \\
\mathbf{3 . 4} \pm \mathbf{0 . 5}^{\mathrm{a}} \\
7.1 \pm 0.6^{\mathrm{b}} \\
9.9 \pm 0.2^{\mathrm{c}}\end{array}$ & $\begin{array}{l}0.3 \\
\mathbf{0 . 4} \\
0.8 \\
1.1\end{array}$ \\
\hline
\end{tabular}

Superscript letters (a-d) indicate (per species per $E$ / Ca) groups that are statistically different (one-way ANOVA).

\subsection{Inter-species differences in element incorporation}

When comparing $\mathrm{Mg}$ incorporation to that of the other elements studied here ( $\mathrm{Ba}, \mathrm{Zn}, \mathrm{Sr}$ and $\mathrm{Na}$ ) between hyaline species (treatment B; Table 3), we observe a positive relation between $D_{\mathrm{Mg}}$ with $D_{\mathrm{Sr}}(p<0.0025), D_{\mathrm{Na}}(p<0.0005)$, $D_{\mathrm{Ba}}(p<0.05)$ and $D_{\mathrm{Zn}}(p<0.005)$. In general, hyaline species are enriched similarly in all elements (Fig. 4). Com- pared to porcelaneous species, the hyaline shell-building species which incorporate most $\mathrm{Mg}$ (>100 $\mathrm{mmol} \mathrm{mol}^{-1}$ $\mathrm{Mg} / \mathrm{Ca}$ ) incorporate more $\mathrm{Na}$ and $\mathrm{Sr}$, while incorporating less $\mathrm{Zn}$ and $\mathrm{Ba}$. Element incorporation across porcelaneous species is less variable than observed for hyaline species. Including data from the literature (both culture and field calibrations; see Table S1 in the Supplement), prefer- 


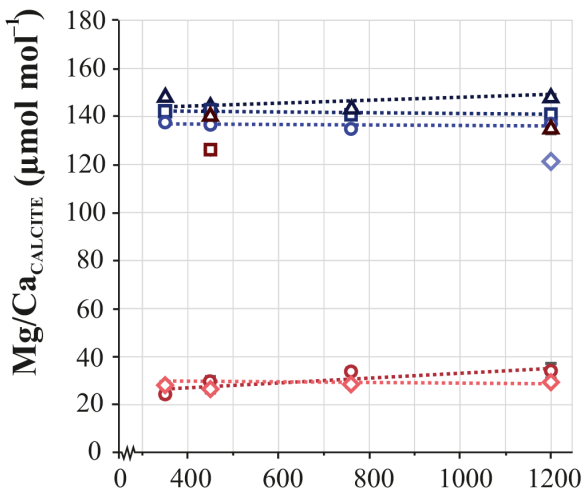

$\Delta$ Sorites marginalis
․ Archaias angulatus
○ Laevipeneroplis bradyi
$\diamond$ Peneroplis pertusus

$\Delta$ Heterostegina antillarum

口 Planorbulina acervali

- Asterigerina carinata

$\diamond$ Amphistegina gibbosa
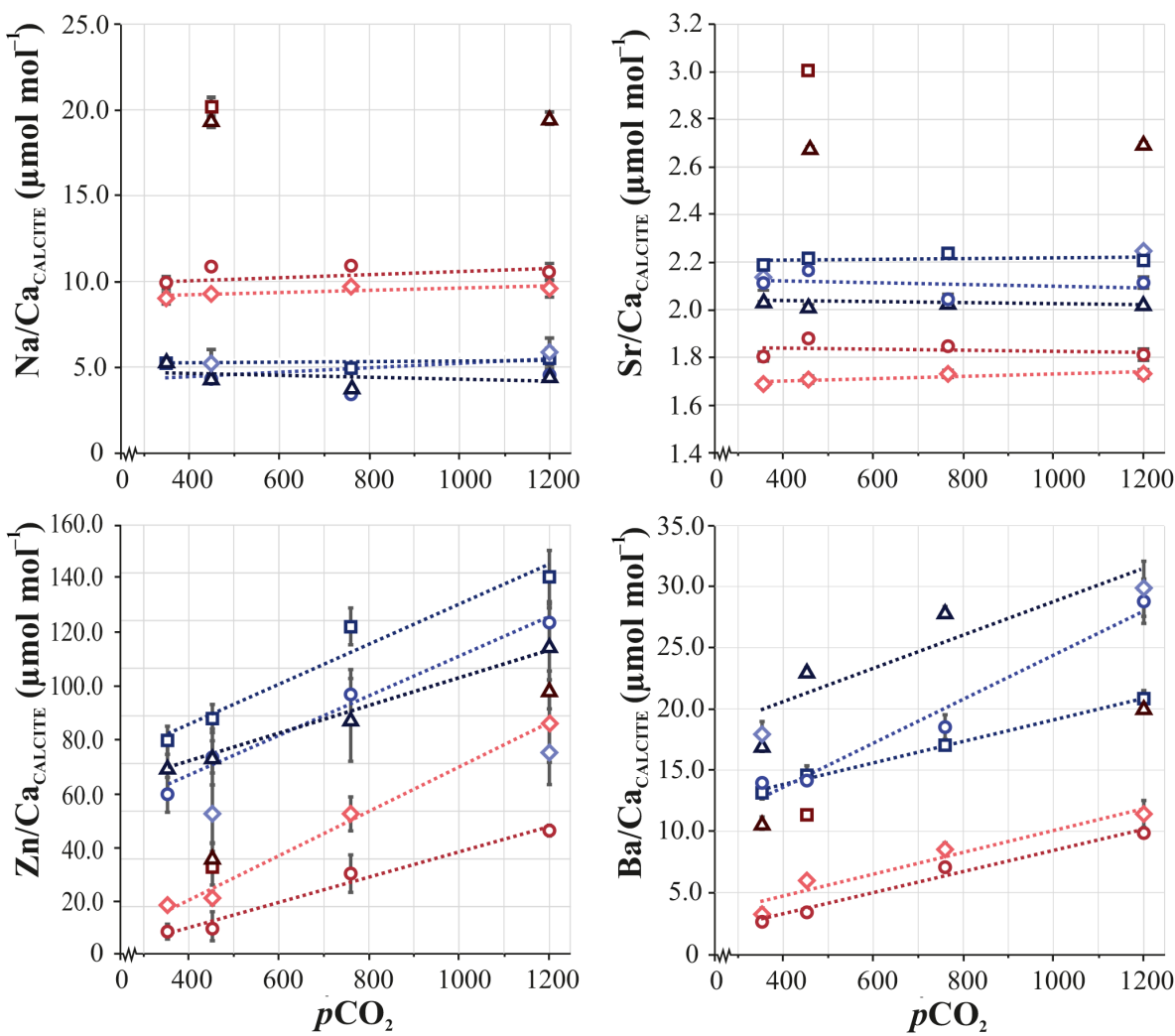

Figure 3. Element to Ca ratios $( \pm \mathrm{SE})$ of different species of foraminifera over a range of $p \mathrm{CO}_{2}$ values. In some cases, the error bar is smaller than the symbol. Porcelaneous species in blue (triangles, S. marginalis; squares, A. angulatus; circles, L. bradyi; squares, P. pertusus) and hyaline species in red (triangles, $H$. antillarum; squares, $P$. acervalis; circles, A. carinata; diamonds, A. gibbosa).

ably those in which both $\mathrm{Mg} / \mathrm{Ca}$ and at least one other element $(\mathrm{Na}, \mathrm{Sr}, \mathrm{Ba}$ or $\mathrm{Zn})$ are measured, shows that the relation based on the Caribbean species studied here is also more generally applicable when including more species $\left(D_{\mathrm{Sr}}=p<0.0025 ; \quad D_{\mathrm{Na}}=p<0.0005\right) ; \quad D_{\mathrm{Ba}}=p<0.005 ;$ $D_{\mathrm{Zn}}=p<0.01$ ), even though these compiled data (labeled "All studies" in Table S2) cover a somewhat wider range in environmental and experiment conditions.

\section{Discussion}

\subsection{Effect of ocean acidification on Element / Ca}

Foraminiferal $\mathrm{Mg} / \mathrm{Ca}, \mathrm{Na} / \mathrm{Ca}$ and $\mathrm{Sr} / \mathrm{Ca}$ do not systematically change with $p \mathrm{CO}_{2}$ for either porcelaneous or hyaline species. The impact of $\mathrm{pH}$ (and/or $\left[\mathrm{CO}_{3}^{2-}\right]$ ) on $\mathrm{Mg} / \mathrm{Ca}$ CALCITE and $\mathrm{Sr} / \mathrm{Ca}_{\text {CALCITE }}$ in foraminifera has been the subject of discussion (e.g., Dissard et al., 2010; Elderfield et al., 1996). In deep-sea benthic species, incorporation of certain elements is governed by carbonate system as observed for $\mathrm{Zn}$ by Marchitto et al. (2005) and Cd 

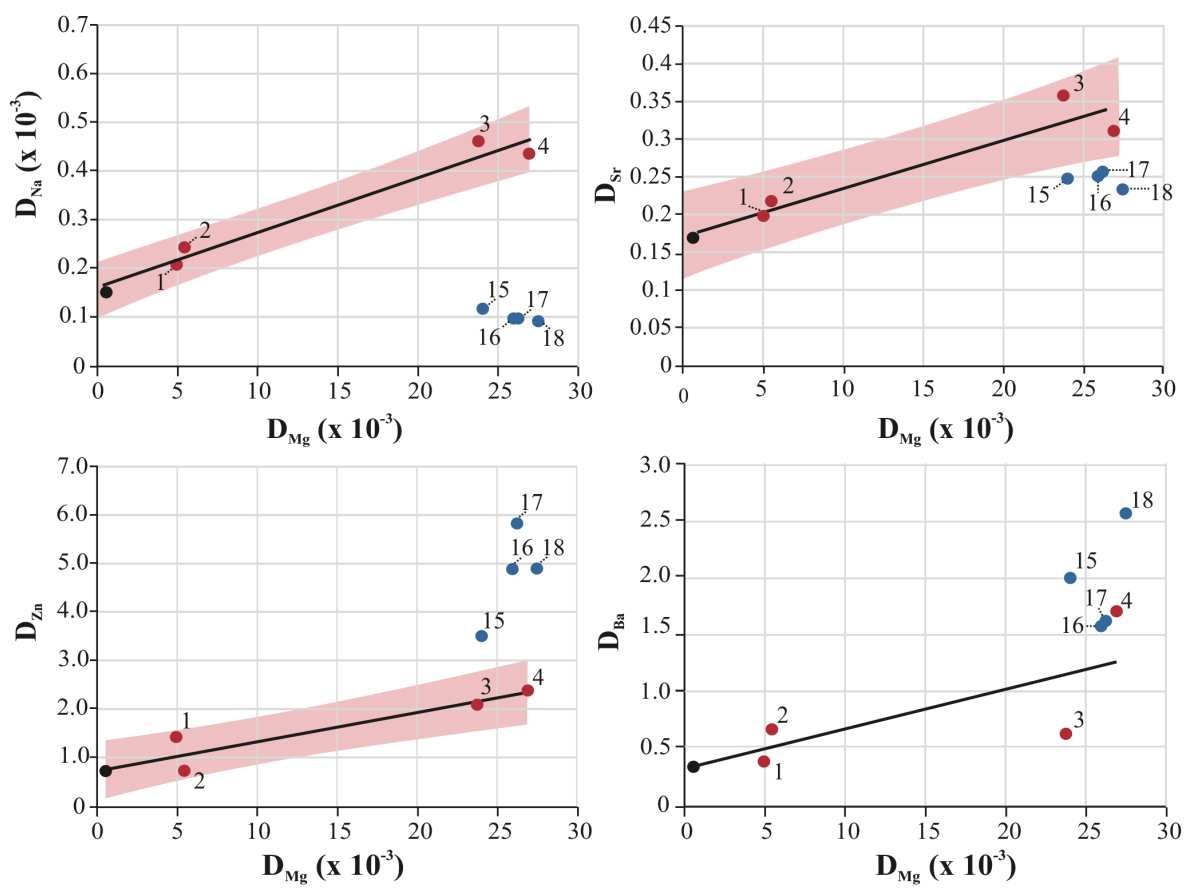

\begin{tabular}{|llcl|}
\hline Hyaline species & $\bullet$ & Milliolid species \\
1 & Amphistegina gibbosa & 15 & Peneroplis pertusus \\
2 & Asterigerina carinata & 16 & Laevipeneroplis bradyi \\
3 & Planorbulina acervalis & 17 & Archaias angulatus \\
4 & Heterostegina antillarum & 18 & Sorites marginalis \\
\hline
\end{tabular}

Figure 4. Partition coefficient of $\mathrm{Na}, \mathrm{Sr}, \mathrm{Zn}$ and Ba versus $D_{\mathrm{Mg}}$ of hyaline (red symbols) and porcelaneous (blue symbols) species. Black lines represent linear trend lines. The $95 \%$ confidence intervals of signification trends $(p<0.025)$ are indicated in red. Black dots represent the NFHS, in-house carbonate standard, consisting of planktonic foraminifera. Numbers correspond to foraminiferal species analyzed; numbers 5-14 and 19-21 represent values from previously published species and are included in Fig. S1 (in the Supplement).

Table 4. Regression and $p$ values of foraminiferal $\mathrm{Zn} / \mathrm{Ca}$ and $\mathrm{Ba} / \mathrm{Ca}$ versus $p \mathrm{CO}_{2}$ values of different species (Fig. 4).

\begin{tabular}{lrr|cr}
\hline & \multicolumn{2}{c|}{$\mathrm{Zn} / \mathrm{Ca}$} & \multicolumn{2}{c}{$\mathrm{Ba} / \mathrm{Ca}$} \\
\cline { 2 - 5 } Species & $R^{2}$ & $p$ value & $R^{2}$ & $p$ value \\
\hline S. marginalis & 0.99 & $<0.0005$ & 0.81 & $<0.025$ \\
A. angulatus & 0.95 & $<0.0025$ & 0.99 & $<0.0005$ \\
L. bradyi & 0.98 & $<0.0005$ & 0.97 & $<0.0025$ \\
A. carinata & 0.98 & $<0.001$ & 0.94 & $<0.005$ \\
A. gibbosa & 0.99 & $<0.0005$ & 0.98 & $<0.001$ \\
\hline
\end{tabular}

and Ba by McCorkle et al. (1995). Observed responses to changes in carbonation ion concentration are in these studies mainly due to calcification in undersaturated seawater, as described by "the carbonate ion saturation hypothesis" (Elderfield et al., 2006). In some low-Mg benthic species, both $\mathrm{Mg} / \mathrm{Ca}$ CAlcite and $\mathrm{Sr} / \mathrm{Ca}$ CAlCiTE do not seem to depend on inorganic carbon system parameters, e.g., $\mathrm{pH}$ or $\left[\mathrm{CO}_{3}^{2-}\right]$ (Allison et al., 2011; Dueñas-Bohórquez et al., 2011). However, for several planktonic species $\mathrm{pH}$ does in- fluence $\mathrm{Mg} / \mathrm{Ca}$ CAlCITE and $\mathrm{Sr} / \mathrm{Ca}$ CAlCiTE (Russell et al., 2004; Lea et al., 1999; Evans et al., 2016). The effect of $\mathrm{pH}$ on $\mathrm{Sr} / \mathrm{Ca}$ CALCITE might be explained via increased growth rates due to $\mathrm{pH}$-associated changes in $\left[\mathrm{CO}_{3}^{2-}\right]$ (Dissard et al., 2010). However, due to the limited experimental setup, we are not able to disentangle the effects of the different carbon parameters in this study. Still, here we show that incorporation of $\mathrm{Mg}, \mathrm{Sr}$ and $\mathrm{Na}$ of the selected larger benthic hyaline and porcelaneous foraminifera is not significantly impacted when cultured over a limited range of $p \mathrm{CO}_{2}$ and thus $\left[\mathrm{CO}_{3}^{2-}\right]$ and $\mathrm{pH}$ values.

In contrast, foraminiferal $\mathrm{Zn} / \mathrm{Ca}$ and $\mathrm{Ba} / \mathrm{Ca}$ are significantly impacted by $p \mathrm{CO}_{2}$ for all species studied here (Table 4; Fig. 3). Although Hönisch et al. (2011) suggested that the impact of carbonate chemistry on $\mathrm{Ba}$ incorporation is negligible, their data do suggest a trend over the same interval in $\mathrm{pH}$ as studied here. In hyaline foraminifera, $\mathrm{Zn} / \mathrm{Ca}$ and $\mathrm{Ba} / \mathrm{Ca}$ increases more as a function of $p \mathrm{CO}_{2}$ (factor of 3.7-4.5 and 3.6-3.7, respectively, when $p \mathrm{CO}_{2}$ increases from 350 to $1200 \mathrm{ppm}$ ) compared to the porcelaneous species (1.3-2.1 and 1.6-2.1 times, respectively). This observation 

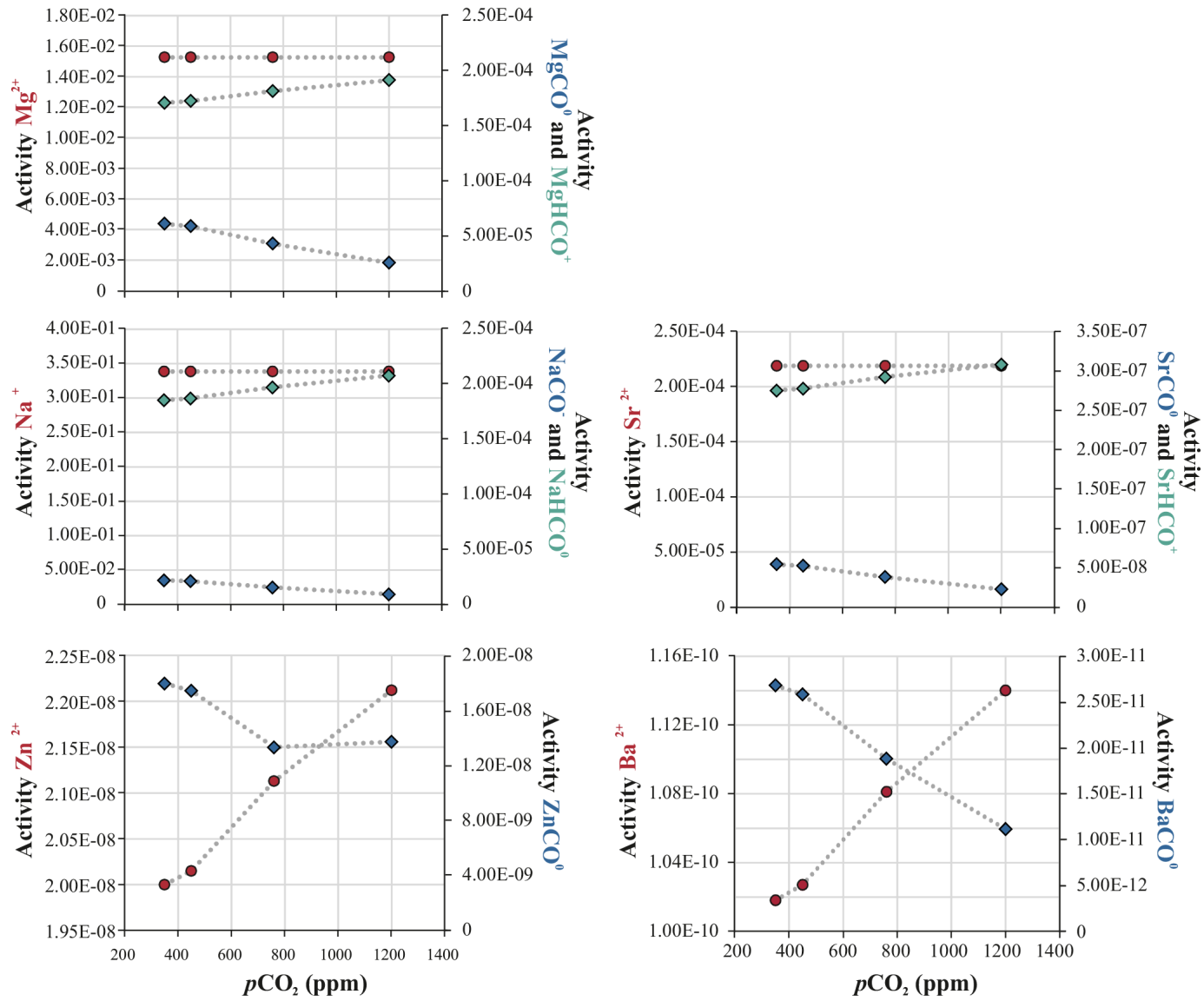

Figure 5. Speciation of $\mathrm{Mg}, \mathrm{Na}, \mathrm{Sr}, \mathrm{Zn}$ and $\mathrm{Ba}$ in the different seawater treatments modeled in PHREEQC (Parkhurst and Appelo, 1999). Activities of free ions (red) and element $(E)$-carbonate complexes $\left(\mathrm{ECO}_{3}\right.$, blue diamonds; $\mathrm{EHCO}_{3}$, green diamonds).

suggests that the mechanisms involved in uptake of $\mathrm{Ba}$ and $\mathrm{Zn}$ are similar for porcelaneous and hyaline species, although the contribution of this process is different, which leads to differences in the sensitivity of $\mathrm{Zn}$ and $\mathrm{Ba}$ incorporation with $p \mathrm{CO}_{2}$

\subsection{Speciation in the microenvironment}

In the culture setup used, increasing $p \mathrm{CO}_{2}$ increases DIC, reduces $\mathrm{pH}$ and thereby decreases seawater $\left[\mathrm{CO}_{3}^{2-}\right]$. Speciation of $\mathrm{Zn}, \mathrm{Ba}$ and also other elements, like $\mathrm{U}$ (Keul et al., 2013; Russell et al., 2004; van Dijk et al., 2017), is primarily controlled by seawater $\left[\mathrm{CO}_{3}^{2-}\right]$. The speciation of all elements studied here ( $\mathrm{Mg}, \mathrm{Na}, \mathrm{Sr}, \mathrm{Zn}$ and $\mathrm{Ba}$ ) for our different seawater treatments was modeled using PHREEQC, a computer program for speciation, batch reaction, onedimensional transport, and inverse geochemical calculations (Parkhurst and Appelo, 1999). For this we used the standard in-software LLNL database, a PHREEQC database that implements most of the inorganic aqueous species and minerals in the thermodynamic data and includes many elements not available in any other PHREEQC database. We observed a decrease in free ions $\left(\mathrm{Zn}^{2+}\right.$ and $\left.\mathrm{Ba}^{2+}\right)$ and an increase in $\mathrm{Ba}$ and $\mathrm{Zn}$ carbonate complexes $\left(\mathrm{BaCO}_{3}^{0}\right.$ and $\left.\mathrm{ZnCO}_{3}^{0}\right)$, with increasing $p \mathrm{CO}_{2}$ (Fig. 5), while the activity of $\mathrm{Mg}^{2+}, \mathrm{Na}^{+}$and $\mathrm{Sr}^{2+}$ remained similar. This suggests that element incorporation in foraminiferal calcite might be depending on the availability of free ions, which in the case of $\mathrm{Ba}$ and $\mathrm{Zn}$ changes with $p \mathrm{CO}_{2}$. This is contrary to inorganic precipitation, where carbonate complexes (e.g., $\mathrm{MgCO}_{3}^{0}$ ) are easily incorporated into the calcite crystal lattice.

\subsection{Trends in element incorporation}

Element incorporation in hyaline foraminifera is highly interdependent, i.e., species with increased $\mathrm{Mg}$ content also incorporate more $\mathrm{Sr}, \mathrm{Na}, \mathrm{Ba}$ and $\mathrm{Zn}$ (Fig. 3). The linear increase in $\mathrm{Sr}$ incorporation with increasing $\mathrm{Mg} / \mathrm{Ca}$ has also been observed for other hyaline species, like Operculina ammonoides (Evans et al., 2015), Amphistegina lessonii and Ammonia aomoriensis (Mewes et al., 2015), which both fall into the same trend as that for inorganic calcite (Mucci and Morse, 1983). Evans et al. (2015) hypothesize that the incorporation of other alkali elements such as $\mathrm{Na}$ is also re- 
(a) $T M T$

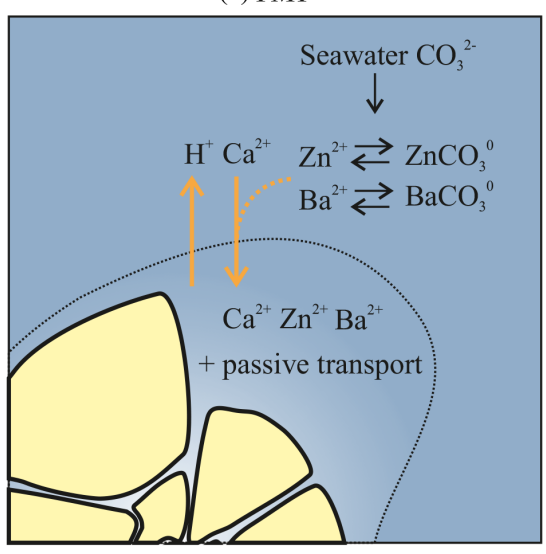

(b) Seawater endocytosis

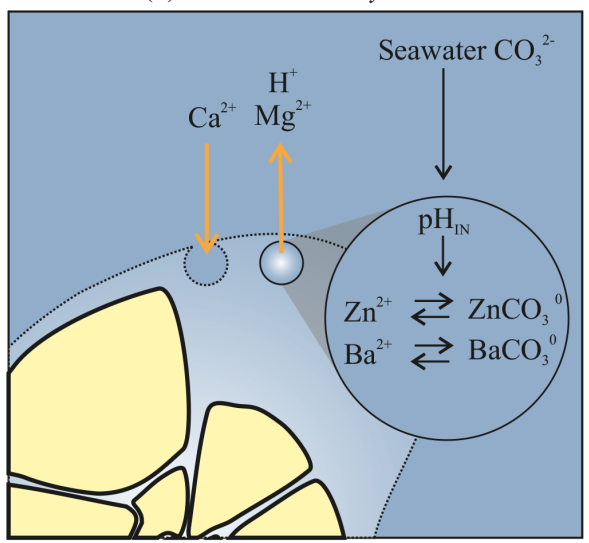

Figure 6. Schematic overview of different mechanisms for ion transport during foraminiferal calcification. Orange arrows indicate transport of ions. (a) The transmembrane transport (TMT) mixing model as proposed by Nehrke et al. (2013). The amount of free ions, e.g., $\mathrm{Zn}^{2+}$ and $\mathrm{Ba}^{2+}$, available for transport by $\mathrm{Ca}^{2+}$ in exchange for protons (Toyofuku et al., 2017) is influenced by speciation due to changes in seawater $\left[\mathrm{CO}_{3}^{2-}\right]$. The composition of the seawater at the site of calcification is determined by both TMT and passive transport. (b) Simplified overview of seawater endocytosis (Bentov et al., 2009; Erez, 2003), where speciation of $\mathrm{Zn}^{2+}$ and $\mathrm{Ba}^{2+}$ is determined by changes in internal $\mathrm{pH}\left(\mathrm{pH}_{\mathrm{IN}}\right)$, which changes with ambient seawater carbonate chemistry. Based on our observations, panel (a) likely applies to hyaline species, whereas panel (b) represents porcelaneous calcification.

lated, due to lattice distortion by the incorporation of $\mathrm{Mg}$, which has been found in inorganic calcite (Okumura and Kitano, 1986). However, although this mechanism might explain some of the observed species-specific element incorporation in hyaline foraminifera, this does not explain the observed difference between hyaline and porcelaneous foraminifera (Fig. 4). Porcelaneous foraminifera have generally high $\mathrm{Mg} / \mathrm{Ca}$, but we actually observe lower incorporation of $\mathrm{Na}$ and $\mathrm{Sr}$ compared to hyaline species with similar $D_{\mathrm{Mg}}$ (Fig. 4, upper right and left panel: $D_{\mathrm{Mg}}$ versus $D_{\mathrm{Na}}$ and $D_{\mathrm{Sr}}$ ). When including porcelaneous species from other studies we also observe no increase in $D_{\mathrm{Sr}}$ over a larger range in $D_{\mathrm{Mg}}$ (Fig. S1, upper left panel: $D_{\mathrm{Mg}}$ versus $D_{\mathrm{Sr}}$ ). Therefore, even though this interdependence might partly stem from mechanisms associated with crystallography, differences between hyaline and porcelaneous foraminifera suggest it could also be caused by mechanisms involved in taking up the ions $\left(\mathrm{Ca}^{2+}\right.$ and $\left.\mathrm{CO}_{3}^{2-}\right)$ necessary for chamber formation, which are different for hyaline and porcelaneous species, reflected in the different trends observed here.

\subsection{Ion transport models}

Both porcelaneous and hyaline foraminifera promote calcification by increasing their internal $\mathrm{pH}$ (De Nooijer et al., 2009). Still, they might use different mechanisms to take up the ions $\left(\mathrm{Ca}^{2+}\right.$ and $\left.\mathrm{CO}_{3}^{2-}\right)$ necessary for chamber formation, which is reflected in the different trends observed here. In both porcelaneous and hyaline foraminifera, $E / \mathrm{Ca}$ responds similarly to changes in $p \mathrm{CO}_{2}$ (Fig. 3), suggesting uptake of all these elements is controlled by the same process. However, we observed different inter-element relations between hyaline and porcelaneous foraminifera (Fig. 4), indicating the mechanisms for ion transport might be different for these two groups. Two of the main concepts of ion transport in foraminifera are transmembrane transport (Nehrke et al., 2013) and the inclusion of seawater by seawater endocytosis (Bentov et al., 2009). Here we try to validate these two concept by comparing them with our observations.

\subsubsection{Transmembrane transport}

During calcification, $\mathrm{Ca}^{2+}$ is proposed to be transported from seawater to the SOC via ion channels (Nehrke et al., 2013), likely in exchange for protons (Toyofuku et al., 2017). This so-called transmembrane transport (TMT) through $\mathrm{Ca}^{2+}$ channels has also been found for other marine organisms, including coccolithophores (Gussone et al., 2006). These $\mathrm{Ca}^{2+}$ channels may not discriminate perfectly between $\mathrm{Ca}$ ions and elements like Mg, Sr, Ba, Na (Sather, 2005; Allen and Sanders, 1994; Hess and Tsien, 1984), causing accidental transport of these elements into the SOC. How much of a certain element will enter the SOC in this way, depends on (1) the selectiveness of the channels and the characteristics of the transported ions (like atomic radius), (2) the element to calcium ratio in the foraminiferal microenvironment and (3) the concentration gradient between seawater and the SOC. The availability of some free ions, like $\mathrm{Ba}$ and $\mathrm{Zn}$, changes as a function of $p \mathrm{CO}_{2}$ due to the formation of carbonate complexes (Fig. 5). When $\mathrm{Zn}$ and $\mathrm{Ba}$ form stable complexes with carbonate ions, they are no longer available for (sporadic) transport through the $\mathrm{Ca}^{2+}$ channels, decreasing the availability at the site of calcification and, subsequently, incorporation into the foraminiferal calcite. Thus, 
transmembrane transport of ions by $\mathrm{Ca}^{2+}$ to the $\mathrm{SOC}$ is in agreement with our results (Fig. 3). The amount of $\mathrm{Zn}$ and $\mathrm{Ba}$ available at the site of calcification is proportional to the concentration of the ratio between $\mathrm{Ca}^{2+}$ and free $\mathrm{Zn}^{2+}$ and $\mathrm{Ba}^{2+}$ in the foraminiferal microenvironment. In turn, the amount of free $\mathrm{Zn}$ and $\mathrm{Ba}$ ions in seawater is controlled by their respective concentration in seawater concentration, as well as carbonate chemistry (Fig. 5). Foraminiferal $\mathrm{Mg} / \mathrm{Ca}, \mathrm{Na} / \mathrm{Ca}$ and $\mathrm{Sr} / \mathrm{Ca}$ is not detectably affected, since the availability of $\mathrm{Mg}^{2+}, \mathrm{Na}^{+}$and $\mathrm{Sr}^{2+}$ does not change over the range of $\left[\mathrm{CO}_{3}^{2-}\right]$ studied here. However, the large range in $\mathrm{Mg} / \mathrm{Ca}$ values in hyaline species suggests that TMT might play a variable role in the calcification process of these species. This may result in an interdependence between all these elements studied such as observed here for the hyaline species if the selectivity for $\mathrm{Ca}^{2+}$ of these channels varies between species.

\subsubsection{Seawater endocytosis}

Another proposed ion transport mechanism is seawater endocytosis (Bentov et al., 2009; Erez, 2003), in which seawater is vacuolized, altered and then used as a calcifying fluid. The chemistry or elemental composition of the vacuolized seawater or the calcification fluid is in this case dependent on the seawater concentration. Inter-species differences would therefore be minimized, as is observed for porcelaneous foraminifera in our study (Fig. 4). However, this concept cannot explain the observed changes in $\mathrm{Zn} / \mathrm{Ca}$ and $\mathrm{Ba} / \mathrm{Ca}$ as a function of $p \mathrm{CO}_{2}$, which are caused by the speciation of elements in seawater due to changes in the carbonate chemistry (Fig. 5). The concentration of total $\mathrm{Zn}$, for example, in the seawater vacuoles does not change for the different treatments, only the species of $\mathrm{Zn}$ present. Only if the internal $\mathrm{pH}$ in the vacuole depends on ambient seawater $\mathrm{pH}$, which is currently unknown, is there a potential for changes in speciation. In theory, if $\mathrm{pH}$ changes in concert with ambient seawater, such a change in internal $\mathrm{pH}$ from $>9$ (De Nooijer et al., 2009) to $>8.6(\Delta \mathrm{pH}=0.4$ in our treatment) will change $\left[\mathrm{CO}_{3}^{2-}\right]$ and thus the speciation of, for example, $\mathrm{Zn}$ (and $\mathrm{Ba}$ ) at the site of calcification. However, over this range the change in $\left[\mathrm{CO}_{3}^{2-}\right]$ will be rather limited and hence such an effect of differential speciation within the calcifying fluid does not suffice to explain the observed sensitivity of $\mathrm{Zn}$ and $\mathrm{Ba}$ to $p \mathrm{CO}_{2}$ in our study. This is in line with recent evidence regarding $\mathrm{Zn} / \mathrm{Ca}$ in foraminifera, which suggests $\mathrm{Zn}$ incorporation is not primarily governed by changes in seawater $\mathrm{pH}$ but by carbonate ion concentration, which does not change much at these high pH's (van Dijk et al., 2017).

\subsection{Consequences for calcification in hyaline and porcelaneous species}

Both ion transport mechanisms, and their consequences for $\mathrm{Zn}$ and $\mathrm{Ba}$ incorporation, are summarized in Fig. 6. The observed species-specific element incorporation in hyaline foraminifera (Fig. 4) is compatible with the transmembrane transport mixing model proposed by Nehrke et al. (2013), where species-specific differences in $E$ / $\mathrm{Ca}$ are explained by the relative contribution of transmembrane transport and socalled passive transport. In contrast to hyaline species, the porcelaneous species show much less inter-species variation in element composition (Fig. 3), suggesting that this group of foraminifera calcifies from a fluid comparable to ambient seawater (Ter Kuile and Erez, 1987) by, for example, seawater endocytosis (Fig. 6, panel b), with only minor alteration of the elemental composition of the calcifying fluid by ion channels. However, the observed correlation between $p \mathrm{CO}_{2}$ and $\mathrm{Ba}$ and $\mathrm{Zn}$ (Fig. 3) suggests that $\mathrm{Ca}$ channels still play a (modest) role in supplying $\mathrm{Ca}^{2+}$ to the porcelaneous SOC, since possible speciation of minor and trace elements in the SOC caused by a change in the internal $\mathrm{pH}$ is probably not sufficient to explain observed patterns (Sect. 4.4.2). However, since porcelaneous species already obtain calcium by including seawater in their calcification vesicle prior to calcite precipitation, contribution of $\mathrm{Ca}^{2+}$ through TMT is likely smaller than in hyaline species, which may explain the observed lower sensitivity of, for example, foraminiferal $\mathrm{Zn} / \mathrm{Ca}$ and $\mathrm{Ba} / \mathrm{Ca}$ to changes in seawater $\left[\mathrm{CO}_{3}^{2-}\right]$ in porcelaneous species (Fig. 3). This approximately 2 times lower sensitivity of porcelaneous foraminifera compared to hyaline species suggests that porcelaneous foraminifera acquire half of the necessary $\mathrm{Ca}^{2+}$ through $\mathrm{Ca}$ channels compared to hyaline species. Element incorporation in porcelaneous foraminifera will therefore be mainly governed by their respective concentrations in seawater, and to a lesser extent by the selectivity for $\mathrm{Ca}^{2+}$ /permeability for other ions during TMT.

\section{Conclusions}

Trends in element incorporation in larger benthic foraminifera can be explained by a combination of differences in calcification strategy and seawater chemistry. Carbonate chemistry of seawater determines speciation and therefore availability of some ions (e.g., $\mathrm{Zn}^{2+}$ and $\mathrm{Ba}^{2+}$ ), which are available for ion transport to the site of calcification. For hyaline foraminifera, we observed species-specific interdependence of element incorporation, which can be explained by a previously proposed transmembrane transport model and the bioavailability of ions in seawater during calcification. For porcelaneous foraminifera, species-specific difference are small, suggesting a higher contribution of another ion source, such as seawater endocytosis.

\section{Data availability}

Data are available and can be requested from the corresponding author (inge.van.dijk@nioz.nl). 


\section{The Supplement related to this article is available online at doi:10.5194/bg-14-497-2017-supplement.}

Competing interests. The authors declare that they have no conflict of interest.

Acknowledgements. This research is funded by the NIOZ - Royal Netherlands Institute for Sea Research and the Darwin Center for Biogeosciences project "Double Trouble: Consequences of Ocean Acidification - Past, Present and Future - Evolutionary changes in calcification mechanisms" and the program of the Netherlands Earth System Science Centre (NESSC). We would like to thank the editor and both reviewers, David Evans and Kazuhiko Fujita, for their constructive comments. Great thanks to Johan Stapel for hosting the 2015 foraminifera culture expedition at the CNSI, St. Eustatius, as well as all the participants: Jelle Bijma and Gernot Nehrke (AWI), Brett Metcalfe (VU), Alice Webb (NIOZ), Esmee Geerken (NIOZ) and Didier de Bakker (NIOZ/IMARES). This study would not have been possible without Steven van Heuven and Bob Koster, who designed and constructed the $\mathrm{pCO}_{2}$ setup (NWO grants 858.14.021 and 858.14.022). Furthermore, we would like to thank Kirsten Kooijman for supplying Dunaliella salina cultures, Patrick Laan and Karel Bakker for seawater analysis, and Mariëtte Wolthers for providing technical support with PHREEQC. Lastly, we thank Jan-Berend Stuut (NIOZ) for the usage of the Hitachi TM3000 SEM (NWO grant 822.01.008 and ERC grant 311152).

Edited by: H. Kitazato

Reviewed by: D. Evans and K. Fujita

\section{References}

Allen, G. J. and Sanders, D.: Two Voltage-Gated, Calcium Release Channels Coreside in the Vacuolar Membrane of Broad Bean Guard Cells, Plant Cell, 6, 685-694, doi:10.1105/tpc.6.5.685, 1994.

Allison, N., Austin, H., Austin, W., and Paterson, D. M.: Effects of seawater $\mathrm{pH}$ and calcification rate on test $\mathrm{Mg} / \mathrm{Ca}$ and $\mathrm{Sr} / \mathrm{Ca}$ in cultured individuals of the benthic, calcitic foraminifera Elphidium williamsoni, Chem. Geol., 289, 171178, doi:10.1016/j.chemgeo.2011.08.001, 2011.

Angell, R. W.: Test morphogenesis (chamber formation) in the foraminifer Spiroloculina hyalina Schulze, J. Foramin. Res., 10, 89-101, 1980.

Barker, S., Higgins, J. A., and Elderfield, H.: The future of the carbon cycle: review, calcification response, ballast and feedback on atmospheric $\mathrm{CO}_{2}$, Philos T. Roy. Soc. A, 361, 1977-1998; discussion 1998-1979, doi:10.1098/rsta.2003.1238, 2003.

Bentov, S. and Erez, J.: Impact of biomineralization processes on the $\mathrm{Mg}$ content of foraminiferal shells: A biological perspective, Geochem. Geophy., Geosy., 7, 1-11, 2006.

Bentov, S., Brownlee, C., and Erez, J.: The role of seawater endocytosis in the biomineralization process in calcareous foraminifera, P. Natl. Acad. Sci. USA, 106, 21500-21504, doi:10.1073/pnas.0906636106, 2009.
Bernhard, J. M., Blanks, J. K., Hintz, C. J., and Chandler, G. T.: Use of the fluorescent calcite marker calcein to label foraminiferal tests, J. Foramin. Res., 34, 96-101, doi:10.2113/0340096, 2004.

Berthold, W.-U.: Biomineralisation bei milioliden Foraminiferen und die Matritzen-Hypothese, Naturwissenschaften, 63, 196197, 1976.

Debenay, J.-P., Guillou, J.-J., Geslin, E., Lesourd, M., and Redois, F.: Processus de cristallisation de plaquettes rhomboédriques à la surface d'un test porcelané de foraminifère actuel, Geobios, 31, 295-302, 1998.

De Nooijer, L. J., Toyofuku, T., and Kitazato, H.: Foraminifera promote calcification by elevating their intracellular pH, P. Natl. Acad. Sci. USA, 106, 15374-15378, doi:10.1073/pnas.0904306106, 2009.

De Nooijer, L. J., Spero, H. J., Erez, J., Bijma, J., and Reichart, G. J.: Biomineralization in perforate foraminifera, Earth-Sci. Rev., 135, 48-58, doi:10.1016/j.earscirev.2014.03.013, 2014.

Dickson, A. G.: Thermodynamics of the dissociation of boric acid in synthetic seawater from 273.15 to $318.15 \mathrm{~K}$, Deep-Sea Res., 37, 755-766, doi:10.1016/0198-0149(90)90004-F, 1990.

Dissard, D., Nehrke, G., Reichart, G. J., Nouet, J., and Bijma, J.: Effect of the fluorescent indicator calcein on $\mathrm{Mg}$ and $\mathrm{Sr}$ incorporation into foraminiferal calcite, Geochem. Geophy., Geosy., 10, 1-13, doi:10.1029/2009GC002417, 2009.

Dissard, D., Nehrke, G., Reichart, G. J., and Bijma, J.: Impact of seawater $p \mathrm{CO}_{2}$ on calcification and $\mathrm{Mg} / \mathrm{Ca}$ and $\mathrm{Sr} / \mathrm{Ca}$ ratios in benthic foraminifera calcite: results from culturing experiments with Ammonia tepida, Biogeosciences, 7, 81-93, doi:10.5194/bg-7-81-2010, 2010.

Dueñas-Bohórquez, A., Raitzsch, M., De Nooijer, L. J., and Reichart, G.-J.: Independent impacts of calcium and carbonate ion concentration on $\mathrm{Mg}$ and $\mathrm{Sr}$ incorporation in cultured benthic foraminifera, Mar. Micropaleontol., 81, 122-130, doi:10.1016/j.marmicro.2011.08.002, 2011.

Elderfield, H. and Ganssen, G.: Past temperature and $\delta^{18} \mathrm{O}$ of surface ocean waters inferred from foraminiferal $\mathrm{Mg} / \mathrm{Ca}$ ratios, Nature, 405, 442-445, 2000.

Elderfield, H., Bertram, C. J., and Erez, J.: A biomineralization model for the incorporation of trace elements into foraminiferal calcium carbonate, Earth Planet. Sci. Lett., 142, 409-423, doi:10.1016/0012-821X(96)00105-7, 1996.

Elderfield, H., Yu, J., Anand, P., Kiefer, T., and Nyland, B.: Calibrations for benthic foraminiferal $\mathrm{Mg} / \mathrm{Ca}$ paleothermometry and the carbonate ion hypothesis, Earth Planet. Sci. Lett., 250, 633649, 2006.

Erez, J.: The source of ions for biomineralization in foraminifera and their implications for paleoceanographic proxies, Rev. Mineral. Geochem., 54, 115-149, doi:10.2113/0540115, 2003.

Evans, D., Erez, J., Oron, S., and Müller, W.: Mg / Ca-temperature and seawater-test chemistry relationships in the shallow-dwelling large benthic foraminifera Operculina ammonoides, Geochimi. Cosmochimi. Ac., 148, 325-342, 2015.

Evans, D., Wade, B. S., Henehan, M., Erez, J., and Müller, W.: Revisiting carbonate chemistry controls on planktic foraminifera $\mathrm{Mg} / \mathrm{Ca}$ : implications for sea surface temperature and hydrology shifts over the Paleocene-Eocene Thermal Maximum and Eocene-Oligocene transition, Clim. Past, 12, 819-835, doi:10.5194/cp-12-819-2016, 2016. 
Guillong, M., Meier, D. L., Allan, M. M., Heinrich, C. A., and Yardley, B. W.: SILLS: A MATLAB-based program for the reduction of laser ablation ICP-MS data of homogeneous materials and inclusions, Mineralogical Association of Canada Short Course Series, 40, 328-333, 2008.

Gussone, N., Langer, G., Thoms, S., Nehrke, G., Eisenhauer, A., Riebesell, U., and Wefer, G.: Cellular calcium pathways and isotope fractionation in Emiliania huxleyi, Geology, 34, 625-628, 2006.

Hemleben, C., Be, A. W. H., Anderson, O. R., and Tuntivate, S.: Test morphology, organic layers and chamber formation of the planktonic foraminifer Globorotalia menardii (d'Orbigny), J. Foramin. Res., 7, 1-25, doi:10.2113/gsjfr.7.1.1, 1977.

Hemleben, C., Erson, O., Berthold, W., and Spindler, M.: fout, Biomineralization in lower plants and animals (edited by: Leadbeater, B.S.C. and Riding, R.) Clarendon Press, Oxford, UK, 237-249, 1986.

Hess, P. and Tsien, R. W.: Mechanism of ion permeation through calcium channels, Nature, 309, 453-456, 1984.

Hönisch, B., Allen, K. A., Russell, A. D., Eggins, S. M., Bijma, J., Spero, H. J., Lea, D. W., and Yu, J.: Planktic foraminifers as recorders of seawater $\mathrm{Ba} / \mathrm{Ca}$, Mar. Micropaleontol., 79, 52-57, 2011.

Jochum, K. P., Scholz, D., Stoll, B., Weis, U., Wilson, S. A., Yang, Q., Schwalb, A., Börner, N., Jacob, D. E., and Andreae, M. O.: Accurate trace element analysis of speleothems and biogenic calcium carbonates by LA-ICP-MS, Chem. Geol., 318-319, 31-44, doi:10.1016/j.chemgeo.2012.05.009, 2012.

Keul, N., Langer, G., De Nooijer, L. J., Nehrke, G., Reichart, G.-J., and Bijma, J.: Incorporation of uranium in benthic foraminiferal calcite reflects seawater carbonate ion concentration, Geochem. Geophy., Geosy., 14, 102-111, doi:10.1029/2012gc004330, 2013.

Kurtarkar, S. R., Saraswat, R., Nigam, R., Banerjee, B., Mallick, R., Naik, D. K., and Singh, D. P.: Assessing the effect of calcein incorporation on physiological processes of benthic foraminifera, Mar. Micropaleontol., 114, 36-45, doi:10.1016/j.marmicro.2014.10.001, 2015.

Langer, G., Sadekov, A., Thoms, S., Keul, N., Nehrke, G., Mewes, A., Greaves, M., Misra, S., Reichart, G.-J., de Nooijer, L. J., Bijma, J., and Elderfield, H.: $\mathrm{Sr}$ partitioning in the benthic foraminifera Ammonia aomoriensis and Amphistegina lessonii, Chem. Geol., 440, 306-312, doi:10.1016/j.chemgeo.2016.07.018, 2016.

Lea, D. W., Mashiotta, T. A., and Spero, H. J.: Controls on magnesium and strontium uptake in planktonic foraminifera determined by live culturing, Geochim. Cosmochim. Ac., 63, 2369-2379, doi:10.1016/S0016-7037(99)00197-0, 1999.

Lear, C. H., Elderfield, H., and Wilson, P. A.: Cenozoic deepSea temperatures and global ice volumes from $\mathrm{Mg} / \mathrm{Ca}$ in benthic foraminiferal calcite, Science, 287, 269-272, doi:10.1126/science.287.5451.269, 2000.

Lueker, T. J., Dickson, A. G., and Keeling, C. D.: Ocean $p \mathrm{CO}_{2}$ calculated from dissolved inorganic carbon, alkalinity, and equations for $\mathrm{K} 1$ and $\mathrm{K} 2$ : validation based on laboratory measurements of $\mathrm{CO}_{2}$ in gas and seawater at equilibrium, Mar. Chem., 70, 105-119, doi:10.1016/S0304-4203(00)00022-0, 2000.
Marchitto, T. M., Curry, W. B., and Oppo, D. W.: Zinc concentrations in benthic foraminifera reflect seawater chemistry, Paleoceanography, 15, 299-306, doi:10.1029/1999PA000420, 2000.

Marchitto, T. M., Lynch-Stieglitz, J., and Hemming, S. R.: Deep Pacific $\mathrm{CaCO}_{3}$ compensation and glacial-interglacial atmospheric $\mathrm{CO}_{2}$, Earth Planet. Sci. Lett., 231, 317-336, doi:10.1016/j.eps1.2004.12.024, 2005.

McCorkle, D. C., Martin, P. A., Lea, D. W., and Klinkhammer, G. P.: Evidence of a dissolution effect on benthic foraminiferal shell chemistry: $\delta^{13} \mathrm{C}, \mathrm{Cd} / \mathrm{Ca}, \mathrm{Ba} / \mathrm{Ca}$, and $\mathrm{Sr} / \mathrm{Ca}$ results from the Ontong Java Plateau, Paleoceanography, 10, 699-714, doi:10.1029/95PA01427, 1995.

Mewes, A., Langer, G., Reichart, G.-J., De Nooijer, L. J., Nehrke, G., and Bijma, J.: The impact of $\mathrm{Mg}$ contents on $\mathrm{Sr}$ partitioning in benthic foraminifers, Chem. Geol., 412, 92-98, doi:10.1016/j.chemgeo.2015.06.026, 2015.

Mezger, E. M., de Nooijer, L. J., Boer, W., Brummer, G. J. A., and Reichart, G. J.: Salinity controls on Na incorporation in Red Sea planktonic foraminifera, Paleoceanography, 31, 1562-1582, doi:10.1002/2016PA003052, 2016.

Mucci, A. and Morse, J. W.: The incorporation of $\mathrm{Mg}^{2+}$ and $\mathrm{Sr}^{2+}$ into calcite overgrowths: influences of growth rate and solution composition, Geochim. Cosmochim. Ac., 47, 217-233, doi:10.1016/0016-7037(83)90135-7, 1983.

Nardelli, M. P., Malferrari, D., Ferretti, A., Bartolini, A., Sabbatini, A., and Negri, A.: Zinc incorporation in the miliolid foraminifer Pseudotriloculina rotunda under laboratory conditions, Mar. Micropaleontol., 126, 42-49, doi:10.1016/j.marmicro.2016.06.001, 2016.

Nehrke, G., Keul, N., Langer, G., de Nooijer, L. J., Bijma, J., and Meibom, A.: A new model for biomineralization and traceelement signatures of Foraminifera tests, Biogeosciences, 10, 6759-6767, doi:10.5194/bg-10-6759-2013, 2013.

Nürnberg, D., Bijma, J., and Hemleben, C.: Assessing the reliability of magnesium in foraminiferal calcite as a proxy for water mass temperatures, Geochim. Cosmochim. Ac., 60, 803-814, doi:10.1016/0016-7037(95)00446-7, 1996.

Okai, T., Suzuki, A., Kawahata, H., Terashima, S., and Imai, N.: Preparation of a New Geological Survey of Japan Geochemical Reference Material: Coral JCp-1, Geostandard. Newslett., 26, 95-99, 2002.

Okumura, M. and Kitano, Y.: Coprecipitation of alkali metal ions with calcium carbonate, Geochim. Cosmochim. Ac., 50, 49-58, doi:10.1016/0016-7037(86)90047-5, 1986.

Parkhurst, D. L. and Appelo, C.: User's guide to PHREEQC (Version 2): A computer program for speciation, batch-reaction, onedimensional transport, and inverse geochemical calculations, US Geol. Surv., Denver, CO, USA, 1999.

Pawlowski, J., Holzmann, M., Berney, C., Fahrni, J., Gooday, A. J., Cedhagen, T., Habura, A., and Bowser, S. S.: The evolution of early Foraminifera, P. Natl. Acad. Sci. USA, 100, 11494-11498, doi:10.1073/pnas.2035132100, 2003.

Pierrot, D., Lewis, E., and Wallace, D. W. R.: MS Excel Program Developed for $\mathrm{CO}_{2}$ System Calculations, Carbon Dioxide Information Analysis Center, Oak Ridge National Laboratory, Oak Ridge, USA, 2006.

Reichart, G.-J., Jorissen, F., Anschutz, P., and Mason, P. R.: Single foraminiferal test chemistry records the marine environment, Geology, 31, 355-358, 2003. 
Russell, A. D., Hönisch, B., Spero, H. J., and Lea, D. W.: Effects of seawater carbonate ion concentration and temperature on shell $\mathrm{U}$, $\mathrm{Mg}$, and $\mathrm{Sr}$ in cultured planktonic foraminifera, Geochim. Cosmochim. Ac., 68, 4347-4361, doi:10.1016/j.gca.2004.03.013, 2004.

Sanyal, A., Hemming, N. G., Broecker, W. S., Lea, D. W., Spero, H. J., and Hanson, G. N.: Oceanic pH control on the boron isotopic composition of foraminifera: Evidence from culture experiments, Paleoceanography, 11, 513-517, 1996.

Sather, W. A.: Selective Permeability of Voltage-Gated Calcium Channels, in: Voltage-Gated Calcium Channels, Springer US, Boston, MA, USA, 205-218, 2005.

Segev, E. and Erez, J.: Effect of $\mathrm{Mg} / \mathrm{Ca}$ ratio in seawater on shell composition in shallow benthic foraminifera, Geochem. Geophy., Geosy., 7, 1-8, doi:10.1029/2005GC000969, 2006.

Ter Kuile, B. and Erez, J.: Uptake of inorganic carbon and internal carbon cycling in symbiont-bearing benthonic foraminifera, Mar. Biol., 94, 499-509, 1987.

Toyofuku, T., Suzuki, M., Suga, H., Sakai, S., Suzuki, A., Ishikawa, T., De Nooijer, L. J., Schiebel, R., Kawahata, H., and Kitazato, $\mathrm{H} .: \mathrm{Mg} / \mathrm{Ca}$ and $\delta^{18} \mathrm{O}$ in the brackish shallow-water benthic foraminifer Ammonia “beccarii”, Mar. Micropaleontol., 78, 113-120, doi:10.1016/j.marmicro.2010.11.003, 2011.

Toyofuku, T., Matsuo, M. Y., de Nooijer, L. J., Nagai, Y., Kawada, S., Fujita, K., Reichart, G.-J., Nomaki, H., Tsuchiya, M., Sakaguchi, H., and Kitazato, H.: Proton pumping accompanies calcification in foraminifera, Nature Communications, Nat. Commun., 8, 14145, doi:10.1038/ncomms14145, 2017. van Dijk, I., de Nooijer, L. J., Wolthers, M., and Reichart, G.J.: Impacts of $\mathrm{pH}$ and $\left[\mathrm{CO}_{3}^{2-}\right]$ on the incorporation of $\mathrm{Zn}$ in foraminiferal calcite, Geochim. Cosmochim. Ac., 197, 263-277, doi:10.1016/j.gca.2016.10.031, 2017.

Wit, J. C., de Nooijer, L. J., Barras, C., Jorissen, F. J., and Reichart, G. J.: A reappraisal of the vital effect in cultured benthic foraminifer Bulimina marginata on $\mathrm{Mg}$ / Ca values: assessing temperature uncertainty relationships, Biogeosciences, 9, 36933704, doi:10.5194/bg-9-3693-2012, 2012.

Wit, J. C., de Nooijer, L. J., Wolthers, M., and Reichart, G. J.: A novel salinity proxy based on $\mathrm{Na}$ incorporation into foraminiferal calcite, Biogeosciences, 10, 6375-6387, doi:10.5194/bg-106375-2013, 2013.

Yu, J. and Elderfield, H.: Benthic foraminiferal $B$ / Ca ratios reflect deep water carbonate saturation state, Earth Planet. Sci. Lett., 258, 73-86, doi:10.1016/j.eps1.2007.03.025, 2007.

Zeebe, R. E. and Sanyal, A.: Comparison of two potential strategies of planktonic foraminifera for house building: $\mathrm{Mg}^{2+}$ or $\mathrm{H}^{+}$removal?, Geochim. Cosmochim. Ac., 66, 1159-1169, doi:10.1016/S0016-7037(01)00852-3, 2002. 